\title{
Control, Models and Industrial Manipulators
}

\section{Erik Hedberg}





\section{Control, Models and Industrial Manipulators}

\section{Erik Hedberg}

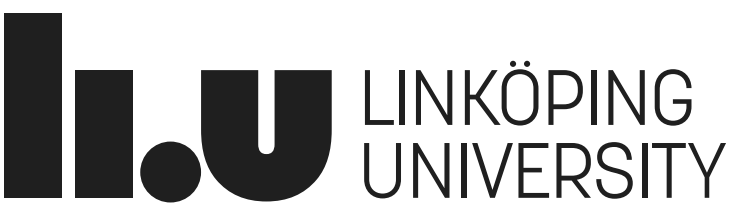


This is a Swedish Licentiate's Thesis.

Swedish postgraduate education leads to a Doctor's degree and/or a Licentiate's degree.

A Doctor's Degree comprises 240 ECTS credits (4 years of full-time studies).

A Licentiate's degree comprises 120 ECTS credits,

of which at least 60 ECTS credits constitute a Licentiate's thesis.

Linköping studies in science and technology. Licentiate Thesis

No. 1894

\title{
Control, Models and Industrial Manipulators
}

\section{Erik Hedberg}

\author{
erik.hedberg@liu.se \\ ww. control.isy.liu.se \\ Department of Electrical Engineering \\ Linköping University \\ SE-581 83 Linköping \\ Sweden
}

ISBN 978-91-7929-740-4 ISSN 0280-7971

Copyright (C 2020 Erik Hedberg

Printed by LiU-Tryck, Linköping, Sweden 2020 




\section{Abstract}

The two topics at the heart of this thesis are how to improve control of industrial manipulators and how to reason about the role of models in automatic control.

On industrial manipulators, two case studies are presented. The first investigates estimation with inertial sensors, and the second compares control by feedback linearization to control based on gain-scheduling.

The contributions on the second topic illustrate the close connection between control and estimation in different ways. A conceptual model of control is introduced, which can be used to emphasize the role of models as well as the human aspect of control engineering. Some observations are made regarding blockdiagram reformulations that illustrate the relation between models, control and inversion. Finally, a suggestion for how the internal model principle, internal model control, disturbance observers and Youla-Kučera parametrization can be introduced in a unified way is presented. 



\section{Populärvetenskaplig sammanfattning}

Arbetet bakom den här avhandlingen grundar sig i två huvudfrågor. Den första handlar om hur styrsystemen för industrirobotar kan förbättras. Den andra handlar om hur man ska tänka kring modellers roll i reglering.

För att bygga en industrirobot med riktigt bra precision behövs både en bra mekanisk konstruktion och ett bra styrsystem som kan reglera dess rörelser. Ett förbättrat styrssystem kan därför ge bättre precision, men också möjliggöra en lättare och mindre kostsam mekanisk konstruktion utan att försämra precisionen.

Två aspekter på robotstyrning undersöks i den här avhandligen. Den första är så kallad skattning, att använda ytterligare sensorer för att få en bättre uppfattning om vad som händer med roboten. I det här fallet så kallade tröghetssensorer, som mäter acceleration och vinkelhastighet. Den andra handlar om reglering, om hur informationen om robotens tillstånd ska användas för att korrigera dess rörelser. Här presenteras ett sätt att jämföra två olika metoder.

Reglerteknik beskrivs ibland som en vetenskap som handlar om att hantera information, och den hanteringen sker oftast i form av så kallade dynamiska modeller, matematiska samband som beskriver hur något förändras över tid.

En av de stora styrkorna med reglerteknik är att man kan använda mätningar från det styrda systemet för att minska behovet av på förhand framtagna modeller. Det leder ibland till att den roll modeller spelar i reglering förbises. I den här avhandligen diskuteras olika exempel som kan användas för att belysa modellkonceptet i reglerteknik. 



\section{Acknowledgments}

This journey has been both sunshine and darkness, and I would like to thank those who have contributed a bit of light along the way.

Martin Enqvist, for the support that kept me going, and for all the discussions. Johan Löfberg, for listening and for seeing what I see. Mikael Norrlöf, for always taking the time to talk, and for the shared train rides. Svante Gunnarsson, for your patience.

Stig Moberg and all the kind people at ABB Robotics, who welcomed me and taught me so much about industrial robotics during lunches and fika breaks.

The fellow PhD students who made life at the division less lonely, both at work and outside of it. Your kindness and friendship have meant a great deal to me.

And of course my family and friends, for your love and support.

Linköping, November 2020

Erik Hedberg 



\section{Contents}

1 Introduction 1

2 Industrial serial manipulators 3

2.1 Definition of industrial serial manipulator . . . . . . . . . . 3

2.2 The market for industrial robots . . . . . . . . . . . 5

2.3 Anatomy of industrial serial manipulators . . . . . . . . . . 7

2.4 Desirable characteristics ............... 10

3 Perspectives on control 13

3.1 Control as motivated action . . . . . . . . . . . . . . 14

3.2 Models and inversion .................. 17

4 From internal model principle to Youla parametrization 23

4.1 Introduction . . . . . . . . . . . . . . 23

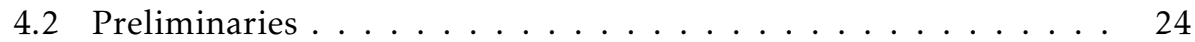

4.3 Proposed procedure . . . . . . . . . . . . . . 27

4.4 Pedagogical merits . . . . . . . . . . . . . . . . . . . . 32

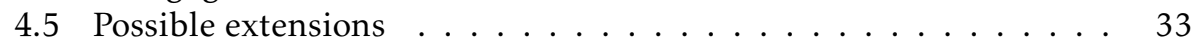

5 Case study: Tool position estimation using inertial measurements $\quad 37$

5.1 Introduction . . . . . . . . . . . . . . . . 37

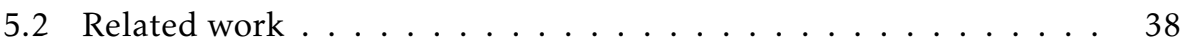

5.3 System overview . . . . . . . . . . . . . . . . . . 38

5.4 Estimation methods . . . . . . . . . . . . . . . . 39

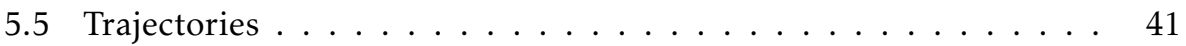

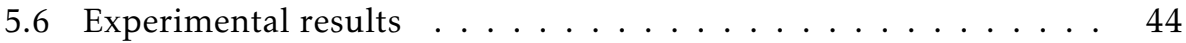

5.7 Discussion . . . . . . . . . . . . . . . . 45

5.8 Conclusions ....................... 46

6 Case study: Feedback linearization compared to LQ-control 47

6.1 Introduction . . . . . . . . . . . . . . . . . 47

6.2 Simulation model . . . . . . . . . . . . . . . . 49

6.3 Control Design . . . . . . . . . . . . . . . 50 


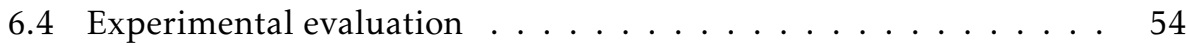

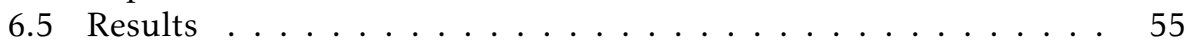

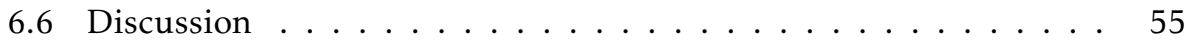

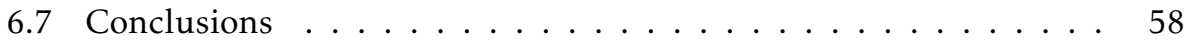

$\begin{array}{ll}\text { Bibliography } & 59\end{array}$ 


\section{1}

\section{Introduction}

Good control systems are fundamental to high-performing industrial manipulators, and good models are fundamental to well-functioning control systems. These two observations motivate the work presented in this thesis.

\section{Industrial manipulators}

Chapter 2 gives a general overview of industrial manipulators. A case study on estimation for industrial manipulators using inertial sensors is presented in Chapter 5, where a complementary filter and a Kalman filter are compared. Chapter 6 compares linear quadratic control design based on Jacobian linearization and feedback linearization respectively.

\section{Models and control}

The great power of feedback is that one can do control without accurate a priori models of the controlled process. Because of this, the role of models in control is sometimes downplayed. Chapter 3 tries to put the role of models in perspective, and offers a discussion on the nature of control as well as some illuminating block diagram reformulations. Chapter 4 offers a suggestion for how the internal model principle, internal model control, disturbance observers and Youla-Kučera parametrization can be taught in a unified way. 



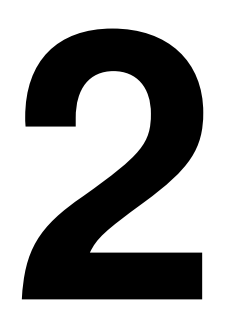

\section{Industrial serial manipulators}

This chapter gives an introduction to industrial robots, in particular to so called serial manipulators. First we introduce some terminology, followed by a brief overview of the market for industrial robots and common applications. Then we discuss how industrial serial manipulators (ISM) are commonly designed, and relate this to what characteristics are desirable for an ISM.

\subsection{Definition of industrial serial manipulator}

The term robot is used to denote many things, from humanoid machines to programmable industrial tools to computer programs.

In this thesis, robot is used in the sense given by ISO 8373 [2012], an international standard entitled Robots and robotic devices - Vocabulary. This standard is endorsed by the International Federation of Robotics (IFR), an industry association counting all major manufacturers of industrial robots as members.

The following definitions are taken from ISO 8373, with minor changes for readability. First the standard defines robot as a physical mechanism.

Robot - Actuated mechanism programmable in two or more axes with a degree of autonomy, moving within its environment, to perform intended tasks.

Then it introduces two main categories, industrial and service robots. ${ }^{1}$

\footnotetext{
${ }^{1}$ An alternative categorization, jokingly used by some industrial robot researchers, is industrial robotics and spectacular robotics.
} 
Industrial robot - Automatically controlled, reprogrammable, multipurpose manipulator, programmable in three or more axes, which can be either fixed in place or mobile for use in industrial automation applications.

Service robot - Robot that performs useful tasks for humans or equipment, excluding industrial automation applications.

In the definition of industrial robot, we see that it is defined as a manipulator, which is also defined by the standard.

Manipulator - Machine in which the mechanism usually consists of a series of segments, jointed or sliding relative to one another, for the purpose of grasping and/or moving objects (pieces or tools) usually in several degrees of freedom.

It is worth noting that in their report World Robotics 2019 - Industrial Robots the IFR, which has been compiling statistics on the robot market for decades, lists several types of machines that would seemingly fit the above definition of industrial robot, but which the IFR does not consider as such for the purposes of their report, see IFR [2019].

Finally, most practitioners likely have little interest in an exact definition of what is to be considered or not an industrial robot, and treat the question on a basis of "I know it when I see it"2.

\subsubsection{Categorization of industrial robots}

The IFR states that "In agreement with the robot suppliers, robots should be classified only by mechanical structure as of 2004", and provides the following definitions.

Articulated robot - A robot whose arm has at least three rotary joints.

Cartesian robot - Robot whose arm has three prismatic joints and whose axes are correlated with a cartesian coordinate system.

SCARA $^{3}$ robot - A robot, which has two parallel rotary joints to provide compliance in a plane.

Parallel/Delta robot $-A$ robot whose arms have concurrent prismatic or rotary joints.

Cylindrical robot - A robot whose axes form a cylindrical coordinate system.

\footnotetext{
${ }^{2}$ A phrase popularized by the US Supreme Court in a case of trying to determine whether a given movie qualified as pornographic or not, see e.g. Gewirtz [1996].

${ }^{3}$ SCARA is sometimes read as Selectively Compliant Articulated Robot Arm, which would then be slightly inconsistent with the above definition of 'articulated' robot.
} 
In this thesis we have chosen to use the term 'industrial serial manipulator' as a reasonable compromise between specificity and convenience. Let us define it as follows.

\section{Definition 2.1. Industrial serial manipulator (ISM)}

An industrial serial manipulator is an articulated industrial robot, consisting of a series of rigid segments connected by actuated rotary joints.

\subsection{The market for industrial robots}

The IFR, as an interest organisation for manufacturers of robots, has as one of its main purposes to collect and compile statistical data from national associations as well as individual member companies. The details in the following section are based on the publicly available parts of the report by IFR [2019].

\subsubsection{Customers and market size}

The automotive sector has long been the largest adopter of industrial robots, although the electronics industry has almost caught up in recent years. This can be seen in Table 2.1, where IFRs categorization of robot installations in 2018 according to industry ${ }^{4}$ is roughly reproduced.

Table 2.1: Share of new robot installations in 2018.

\begin{tabular}{lc} 
Industry & Share \\
\hline Automotive & $30 \%$ \\
Electronics & $25 \%$ \\
Metal and machinery & $10 \%$ \\
Plastic and chemical & $5 \%$ \\
Food & $2 \%$ \\
Others & $10 \%$ \\
Unspecified & $18 \%$ \\
\hline
\end{tabular}

According to the aforementioned report, around 420000 industrial robots were installed during 2018, an increase of 6\% compared to 2017. The number of new installations is reported to have grown on average 19\% yearly between 2013 and 2018.

Using an estimated average robot service life of 12 years $^{5}$, the IFR calculates that there are currently 2.7 million industrial robots deployed worldwide, based on

\footnotetext{
${ }^{4}$ Based on the wonderfully named International Standard Industrial Classification of All Economic Activities (ISIC), maintained by the Statistics Division of the United Nations (UNSD).

${ }^{5}$ Based on a study carried out in 2000 by the United Nations Economic Commission for Europe together with the IFR.
} 
previously reported installations. The report however also notes that there are some indications that this service life estimate could be too conservative, and that some tax authorities on the other hand calculate with a service life of five years.

\subsubsection{Common applications}

The perhaps most common view of industrial robots among the public is as replacement for human labor, but to a large degree they are also a replacement for other machines with a narrower range of applications. While industrial robots might require a larger initial investment, their multipurpose nature can allow for faster and cheaper reconfiguration of production lines, or even enable manufacturing of heterogeneous product models on a single production line.

Industrial robots are used for a wide variety of tasks, as exemplified by the detailed classification of applications used by the IFR, briefly summarized below:

- Handling and machine tending, such as palletizing, packaging, picking and placing, metal casting, plastic moulding, inspection and testing.

- Welding and soldering, such as arc-, laser-, ultrasonic-, or plasma-welding.

- Dispensing, such as painting, sealing and coating.

- Processing, such as laser or water cutting, grinding, milling and polishing.

- Assembling and disassembling.

- Cleanroom use, for flat panel displays and semiconductors.

The flexibility to tackle such a wide variety of tasks comes not only from a versatile mechanical structure and exchangeable tools, but also from software that makes it easy for the customer to program the robot for various use cases.

\subsubsection{Software}

All major robot manufacturers offer software packages both for facilitating general deployment, programming and operation of robots and to provide specialized functionality tailored to certain applications.

In addition to programming the motion of individual robots, it is also important to provide software that support the design, visualization and simulation of entire production cells where robots and other machines work in synchronization.

Some examples of applications for which manufacturers offer specific software packages are picking and placing of items on conveyor belts, spot welding, laser cutting, palletizing and painting. 


\subsection{Anatomy of industrial serial manipulators}

As previously defined, a serial manipulator consists of a series of more or less rigid segments, referred to as links, connected by rotary joints.

\subsubsection{Joint configurations}

The number of joints determine the degrees of freedom (DOF) of the manipulator, which in turn is an upper limit on the degrees of freedom with which the tool at the end of the manipulator can be positioned.

Thus, six degrees of freedom are required to freely choose position and orientation, collectively referred to as pose, of the tool, and therefore most ISMs feature six joints. The point on the tool for which the position is specified is usually called the tool center point (TCP). Some applications do not require full 6 DOF positioning, e.g., when stacking products on a pallet it is often enough to only control position and orientation around the vertical axis.

The most common configuration of the first three joints is the so-called elbowconfiguration, where the first joint rotates around the vertical axis and the two subsequent joints have axes of rotation that are parallel to each other and to the horizontal plane.

For 6-DOF manipulators, the last three joints are almost always in a so-called spherical wrist configuration. This means that their axes of rotation always intersect in a common point, referred to as the wrist center point (WCP).

The main appeal of the spherical wrist configuration is that it simplifies the socalled inverse kinematics problem of finding joint angles corresponding to a desired TCP pose. One can first use the orientation to solve for the 'wrist angles', and then the position to solve for the 'elbow angles'.

\subsubsection{Physical characteristics}

The size of the links, together with the placement of the joints, determines which end-effector poses are attainable, and the set of such poses is called the robot workspace. Often the (maximum) reach of the robot is used as a proxy for the size of the workspace. Most industrial robots have a reach in the range of 0.5 to 4 meters.

The maximum load that a robot model can lift while sustaining a given level of performance is often referred to as the (maximum) payload of that model. Most major robot manufacturers offer models with payloads in the range of 3 to 600 $\mathrm{kg}$, with some offering maximum payloads as high as 1500 or even $2300 \mathrm{~kg}$.

The links are most often constructed out of metal to make sure that the structure is as rigid as possible. In so-called collaborative robots, where safety dictates that the kinetic energy of the links be limited, plastic is often used to reduce the weight of the links. 
Table 2.2: Range of maximum payload and reach for industrial serial manipulators, as listed online by manufacturers. Definitions of reach and payload vary between manufacturers, so numbers should be considered as approximate. Data collected from manufacturer websites during summer of 2020.

\begin{tabular}{lrrr}
\hline Manufacturer & Payload $[\mathrm{kg}]$ & Reach $[\mathrm{m}]$ & Company origin \\
\hline Fanuc & $4-2300$ & $0.55-4.7$ & Japan \\
Kawasaki & $3-1500$ & $0.5-4$ & Japan \\
KUKA & $2-1200$ & $0.5-4$ & Germany \\
Nachi & $1-1000$ & $0.35-4$ & Japan \\
ABB & $0.5-800$ & $0.48-4.2$ & Sweden \\
Yaskawa & $0.5-800$ & $0.35-4$ & Japan \\
Comau & $3-650$ & $0.63-3.7$ & Italy \\
Hyundai & $6-600$ & $0.93-3.5$ & South Korea \\
Stäubli & $2.3-190$ & $0.52-3.7$ & Switzerland \\
Panasonic & $4-22$ & $1.1-2.7$ & Japan \\
Shibuara Machines & $3-20$ & $0.6-1.7$ & Japan \\
Yamaha & $1-20$ & $0.9-1.5$ & Japan \\
Universal Robotics & $3-16$ & $0.5-1.3$ & Denmark \\
Doosan & $6-15$ & $0.9-1.7$ & South Korea \\
Omron & $4-14$ & $0.65-1.3$ & Japan \\
Mitsubishi & $3-12$ & $0.5-1.4$ & Japan \\
Epson & $2.5-12$ & $0.45-1.4$ & Japan \\
Denso & $0.5-12$ & $0.43-1.3$ & Japan \\
\hline
\end{tabular}

There are a few industrial robot models where the links are partly manufactured from carbon fiber to decrease weight while maintaining high stiffness. Carbon fiber is however less favourable in other respects such as cost and durability, limiting its success as a material for industrial robots.

Table 2.2 lists some manufacturers of industrial serial manipulators, and the available range of maximum payload and reach in the models presented on the company website. The companies that offer robots with payloads above $100 \mathrm{~kg}$ can be considered prominent manufacturers of traditional industrial robots. Universal Robotics is a well-known manufacturer of collaborative robots.

\subsubsection{Actuation}

Modern robots are practically always actuated by electric motors, and in general the control of the electrical current that generates the motor torque is fast enough that the motors can, for most purposes, be approximated as generating instantaneous torque.

The most straightforward way of actuating all joints is to place a motor at each joint. However, keeping the mass and the amount of cabling at the outer links low is usually advantageous for performance. Therefore it is not uncommon to 
supply mechanical power for subsequent joints by some transmission mechanism built into a link.

In general, industrial robots use a geared transmission between motors the and subsequent links, with gear ratios commonly in the double or triple digit region. Rarely are joints actuated with so-called direct drive, where one revolution of the motor is equal to one revolution of the actuated joint.

A higher gear ratio offers several advantages, most notably it allows for larger torques and better precision. The major drawback is that gearboxes invariably introduces additional flexibility into the manipulator, as well as friction and backlash. For this reason good gearboxes, and good models of them, are crucial for high-performing manipulators.

\subsubsection{Sensors}

To estimate the position of the end-effector, most industrial robots use only measurements of the motor angles, which by virtue of the high gear ratio gives good static precision but requires accurate gearbox models to estimate the dynamic behaviour correctly. There are some commercial offerings that use additional sensors, such as a secondary encoder to directly measure the arm angle, i.e. the angle after the gearbox, or an inertial measurement unit (IMU) to measure vibrations and adjust the program to reduce them. In applications where the tool needs to apply pressure to a work object, force sensors are sometimes installed as a part of the tool.

For an academic robot researcher, additional sensors hold the promise of increased control performance. For an industrial robot designer, more sensors also mean higher cost, additional design complexity and more components that can malfunction.

\subsubsection{Control cabinet}

It is common to place part of the robot control system and power supply electronics in a separate cabinet. Such cabinets can often control several robots as well as other machines. One benefit of this separation is that placing the cabinet out of reach from the robot assures it can be safely accessed.

\subsubsection{Specializations}

One example of adaptation for specific industry is the use of food-grade oil in robots for use in food-processing, such that any leakage does not contaminate the products. Another is robots for spray painting, which need to be carefully sealed so that paint does not enter sensitive parts of the robot as well as built so that the risk of causing sparks that can ignite the aerosol paint is minimized. 


\subsection{Desirable characteristics}

The wide variety of tasks for which robots are used require designers to consider many aspects of what it means for a robot model to perform well.

\subsubsection{Motion performance}

The most obvious criteria for a good industrial robot is that it should closely follow the movements we specify.

To get a feel for how such motion performance is measured, it is instructive to look at the standard ISO 9283 [1998], Manipulating industrial robots — Performance criteria and related test methods, which specifies the following performance characteristics:

- Pose accuracy

- Pose repeatability

- Multi-directional pose accuracy variation

- Distance accuracy

- Distance repeatability

- Position stabilization time

- Position overshoot

- Drift of pose characteristics
- Exchangeability

- Path accuracy

- Path repeatability

- Path accuracy on reorientation

- Cornering deviations

- Path velocity characteristics

- Minimum posing time

- Static compliance

- Weaving deviations

Accuracy means that the result is close to what is specified in the program, while repeatability means that the result is similar every time the programmed action is performed, as illustrated in Figure 2.1.

Accuracy can be relative to a given TCP position or relative to the base of the robot, and by extension to the 'world frame'. The former can be referred to as relative accuracy and the latter as absolute accuracy. Lack of accuracy can in some applications easily be compensated with manual adjustment, while in others it is critical that the tool can be positioned accurately based solely on coordinates supplied programmatically.

Repeatability is in general rather good with most industrial manipulators. In a brief survey of online catalogues, manufacturers claim static repeatability between 0.05 and $0.5 \mathrm{~mm}$ for most of their models. Static repeatability is mainly a property of the mechanical construction, and can hardly be improved by a control system without additional sensors.

In contrast, good accuracy requires careful calibration and software compensation. Measures of accuracy rarely seem to figure in advertising material, and 


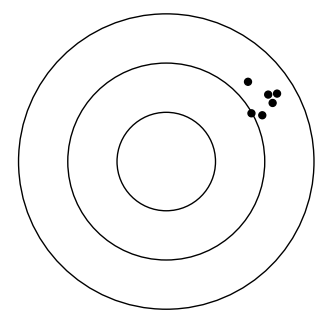

(a) Low accuracy, good repeatability

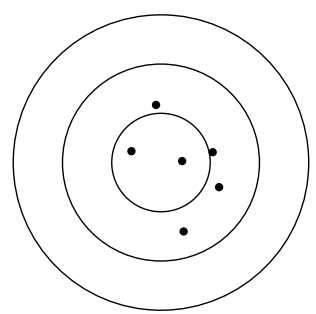

(b) Better accuracy, worse repeatability

Figure 2.1: Some applications require good repeatability while other need high accuracy. If accuracy is measured in relation to another position of the end-effector we call it relative accuracy, if it is in relation to the robot base we call it absolute accuracy.

many suppliers offer additional services or solutions for customers who require high accuracy.

\subsubsection{Other characteristics}

\section{Cost}

The cost to purchase a manipulator is of course a crucial factor for commercial success, it was for example reported as the biggest barrier to robot adoption in a survey of 85 companies across various industries, see McKinsey \& Company [2019]. From an engineering perspective, the cost of manufacturing a manipulator is closely related to the quality of the control system, as good motion control will to some degree allow for a less expensive mechanical construction without loosing performance.

\section{Ease of use}

Configuring and programming robots can be quite time consuming, and ease of use is a characteristic that has a direct and non-negligible impact on the total cost of installing and operating a robot.

\section{Robustness}

A good industrial robot design should be robust, in a broad sense of the word, to make it cost-efficient to manufacture and to allow users to obtain good performance without expert knowledge.

For example it should ideally be robust with regards to manufacturing tolerances in the mechanical components, to wear in said components over time, to the way it has been mounted, to additional cabling mounted on the links, to changes in temperature, to imperfect load information and to suboptimal tuning. 
Finding a good balance between best case performance and robustness is an important consideration, especially in designing the control system of the robot.

\section{Safety}

Safety is both important and difficult, but easily forgotten in an academic setting. When installing and operating robots, substantial effort is required to ensure that the risk of human injury is minimized.

Safety is primarily achieved by fencing off the robot cell and making sure that the robot stops whenever anyone enters the cell. But if the robot system itself can be made safer, the need for costly external safety measures and procedures is reduced. This is one of the key selling points of so-called collaborative robots, or 'cobots', which are meant to work alongside human workers without requiring extra safety measures. 


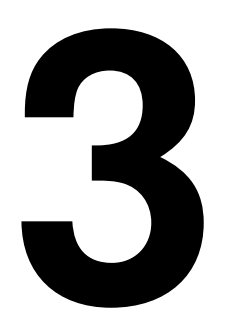

\section{Perspectives on control}

This chapter introduces a model of control which can be used to emphasize the role of models and the human aspect in control engineering, as well as some block diagram reformulations that can be used to illustrate the relation between models, control and inversion.

Readers looking for an introduction to automatic control are well served by the textbook Feedback Systems by Åström and Murray [2008], a modern text with emphasis on fundamental concepts, also freely available from the authors' website. A more traditional as well as comprehensive, but equally solid and insightful, textbook is Control System Design by Goodwin et al. [2001]. The tutorial article Feedback for physicists by Bechhoefer [2005] offers a concise but rich introduction.
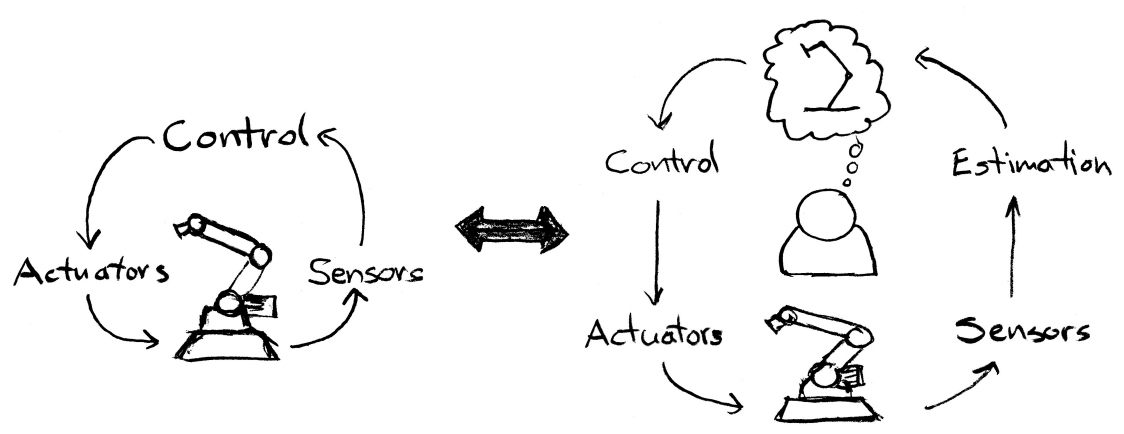

Figure 3.1: The word control can mean several things. At the heart of every control loop are the beliefs and desires of the designer. 


\subsection{Control as motivated action}

\subsubsection{Definition}

The following four definitions together form a simple model that gives a possible definition of "control" as an activity and the main characteristics of that activity.

Definition 3.1 (Control).

Performing control means taking motivated action.

\section{Definition 3.2 (Motivated action).}

An action is motivated if it is based on a desire, and on a belief of how the action will affect the fulfillment of the desire. ${ }^{1}$

Definition 3.3 (Desire).

A desire is a combination of a model and a measure of similarity.

\section{Definition 3.4 (Belief).}

A belief is a combination of a model and a degree of confidence.

Automatic control, which we might also call engineered control (or perhaps artificial control, in keeping with the times), could then be defined as the art and science of encoding beliefs and desires into a decision making mechanism.

This model, which we could call the desire-belief model, might seem too broad or too toy-like to be of any use in an engineering context. I believe however that, thanks to its compactness, it can serve as a tool for highlighting two important aspects of automatic control when discussing or teaching. And to quote Ljung [1999], our acceptance of models should be guided by "usefulness" rather than "truth".

First, with the choice of words it emphasizes the human component in control. Second, it emphasizes the role of models, especially the fact that models are used to represent both what we want and what we think we know.

\subsubsection{The human component}

In the above definition, the words desire and belief are chosen because they have a decidedly human ring to them. The purpose of this is to emphasize the inevitable human component in control engineering, a perspective that is easier to overlook when using words with a more technical flavour, such as objective or model.

\footnotetext{
${ }^{1}$ This definition is taken more or less directly from the so called Humean theory of motivation, due originally to David Hume. See e.g. Smith [1987].
} 
One can argue whether machines are capable of harbouring desires and beliefs, but as long as control is a human endeavour, human desire and belief will be an inevitable part of control.

Take for example the design of an industrial manipulator with automatic motion control. No matter how ingenious the control design is, there will always exist trade-offs between different aspects of system performance. And to decide what balance best suits the customer's desires requires an operator with an understanding both of the customer's needs and of the industrial manipulator.

This example also an illustrates the multi-layered nature of control, where the operator is part of a tuning process that controls the robot control system. Using the broad definition of control introduced previously, one can frame every automatic controller as being part of a larger control process, which is in turn part of an even larger process et cetera. The innermost processes might be well described by technical models, but as the perspective broadens a purely technical description becomes less and less feasible.

In the above the definition of belief the human component is also illustrated by the division into model and confidence. It emphasizes the fact that no matter how well our models perform in experiment, in the end we have to use our judgment to decide how confident we are in the model.

One could argue that our confidence in the model could be regarded as part of the model, perhaps quantified by some measure of probability. But by clearly separating the concept of a plant model, which might be probabilistic or not, and our confidence in it, the role of human judgment in automatic control is highlighted.

Ultimately this can be related to a fundamental problem in philosophy of science, the problem of induction, described in e.g. Henderson [2020]. In short, how can we be sure that the sun will rise tomorrow? Since we cannot formally prove that it will, our certainty that it will indeed rise has to come in some part from human judgment, and not solely from formal reasoning.

The division of desire into model and similarity tries to capture the fact that it can sometimes be quite straightforward to describe the result one would ideally like to have, but more difficult to rank suboptimal results in order of desirability. This leads to situations where some degree of the desired behaviour can be captured by formal specifications such as objective functions and mathematical models, but where the final measure of desirability is expressed through the judgment of a skilled operator tuning the control system.

\subsubsection{The dual role of models}

The second benefit of using the proposed definition of control is that it emphasizes the two-fold role of models; they express both what we believe about the system and how we would like it to behave ${ }^{2}$.

\footnotetext{
${ }^{2}$ And as Willems [2007] would phrase it, the behaviour is all there is.
} 
These two roles and their interplay is efficiently illustrated by the verb "to expect". Consider the following sentence:

"I expect this to be done by tomorrow."

This can be interpreted as an estimate, an answer to the question when something will be done. But it can equally be seen as a demand, as a command from someone who wants something done by tomorrow.

Especially the latter interpretation is interesting from a control perspective, as upon further consideration it is not purely a demand. When a proficient manager tells her subordinate "I expect you to finish by tomorrow", it is part demand and part estimate; the statement expresses a belief that the employee is capable of finishing the task by tomorrow.

In an ideal case, we can represent these two roles by two separate structures, as depicted in Figure 3.2. Here the first structure contains a model of our desire, and feeds a suitable representation of it into the second structure, which contains our belief of how to choose the plant input in order to get the desired output, i.e. to "invert" the plant.

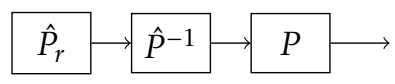

Figure 3.2: In an ideal control scenario, we have a model of what we want, $\hat{P}_{r}$, and an other model, $\hat{P}^{-1}$, of how to invert the plant $P$ perfectly.

Note that in such an ideal scenario, if our desire for the system behaviour changes, we would only have to change parameters in the first structure to reflect this. However, such an ideal separation is rarely possible since it would require perfect inversion.

If we have to approximate the inversion, the choice of approximation will impact the system behaviour. It should thus be chosen to reflect our desire as well as our belief. Since in practice all inverses of dynamical systems are approximate, this point is important to keep in mind when interpreting control schemes that involve explicit plant models.

One example is the so called internal model control (IMC) scheme, depicted in Figure 3.3. At a first glance, it might look like there is a clear division of roles, where $\hat{P}$ represents our belief of how the plant functions while the parameters representing our desired system behaviour would be in $Q$. But upon further consideration it is not certain that a more accurate ${ }^{3}$ model $\hat{P}$ in this scheme will yield a more desirable behaviour for a given $Q$.

When a model is used to predict the plant behaviour, and the controller is fashioned to counteract all unexpected behaviour, the result is not only rejection of

\footnotetext{
${ }^{3}$ We have not touched upon the question of how to measure or rank the correctness of various beliefs, but arguably the measure of similarity used to compare actual behaviour to desired behaviour can also be used to compare actual behaviour to predicted behaviour.
} 


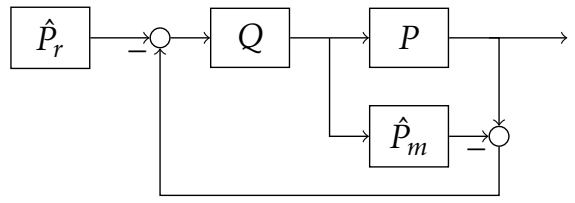

Figure 3.3: The so called internal model control scheme, here depicted with an explicit model for the reference signal.

exogenous disturbances but also an element of model following. The controller is in a sense trying to suppress the differences between how the plant and the model respond to inputs, whether this difference is a result of exogenous (unmodeled) disturbances or of incorrectly modeled plant dynamics.

Thus by using an incorrect model inside the feedback loop, the plant behaviour can be shifted towards that of the model. If the model is used outside of the feedback loop, as illustrated in Figure 3.4, there is model following in the sense of tracking the model output.

Emphasizing this dual role of models is also a question of emphasizing the role of models in general. In feedforward control, where the controller does not receive measurements from the controlled process, it is clear that explicit models for the process are needed.

The role of models in feedback control is less obvious, and indeed one of the main benefits of feedback control is that it reduces the need for a priori models. Because of this, the role of models in feedback control is sometimes downplayed, with some researchers even labeling their methods as "model-free control", like Fliess and Join $[2013]^{4}$.

One could take the view that the fundamental need for models is not reduced in feedback control, but rather that the burden is shifted from using a "synthetic" model to using the actual plant as a "real" model. This perspective is illustrated in the following section.

\subsection{Models and inversion}

In the previous discussion, the term model was used in a rather general sense. In automatic control terminology, a model is typically understood to mean a representation of the (causal) input-to-output relation, while a representation that describes the (non-causal) relation output-to-input is referred to as an inverse model. The former is sometimes referred to as a forward model for the sake of clarity, while the later is sometimes just called an inverse.

It is often pointed out that the concept of inversion is at the heart of automatic

\footnotetext{
${ }^{4}$ Although they describe their method by stating that "the unknown 'complex' mathematical model is replaced by an ultra-local model".
} 

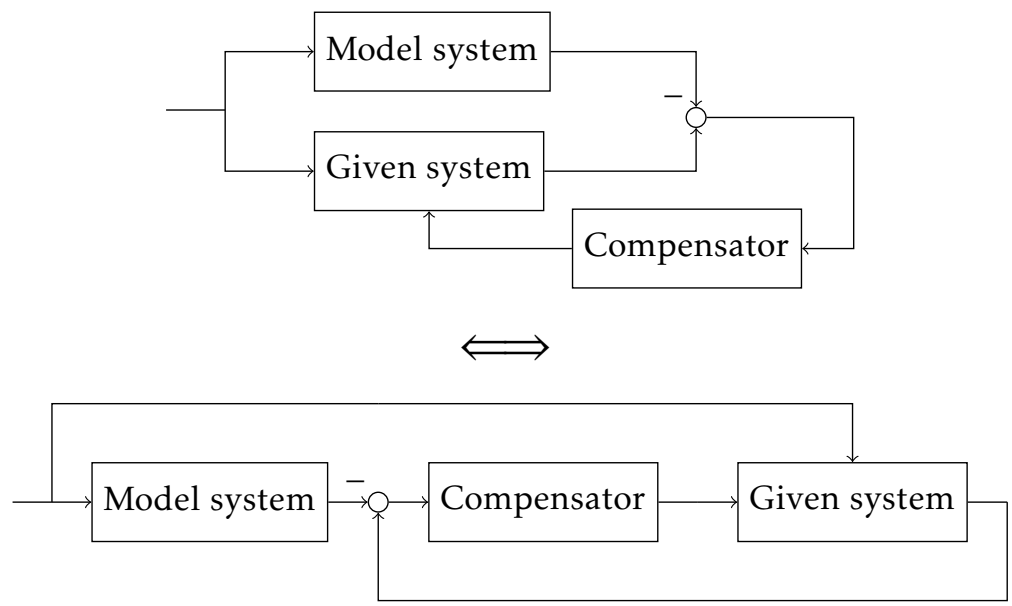

Figure 3.4: A "model following control system", at the top as depicted in the textbook by Wolovich [1974], and at the bottom in a perhaps more familiar "feedforward form".

control, and that an approximate inverse can be obtained by using feedback from a forward model, see e.g. Chapter 2 in Goodwin et al. [2001].

\subsubsection{Complementary filter interpretation of IMC}

A corollary of this is that any standard error feedback control loop can be reformulated as feedforward control where a perfect model is used in the approximate inverse. This, together with a way of mixing the two formulations, is illustrated in Figure 3.5.

This "mixing" structure, a so called complementary filter, where the two blocks $H$ and $1-H$ sum to unity, becomes interesting when we consider the use of an imperfect forward model $\hat{P}$. In that case, which is illustrated in the top diagram of Figure 3.6, the complementary filter weights together a measured output from the true plant $P$ and a predicted output from the model $\hat{P}$.

This can be compared to the reformulation of a state-space observer as two transfer functions, one from the input to the estimate, $T_{1}$, and one from the output to the estimate, $T_{2}$. The estimate is then unbiased in the sense that

$$
C\left(T_{1}+T_{2} \hat{P}\right)=\hat{P}
$$

where $C$ is the state-to-output mapping of the state space form of $\hat{P}$. For details see e.g. Goodwin et al. [2001]. In this comparison, $T_{2}$ would correspond to $H$ and $T_{1}$ to $(1-H) \hat{P}$.

More interesting to note however, is how straightforward it is to transform this complementary filter scheme into an equivalent internal model control scheme, following the steps depicted in Figure 3.6. This offers an intuitive interpretation 


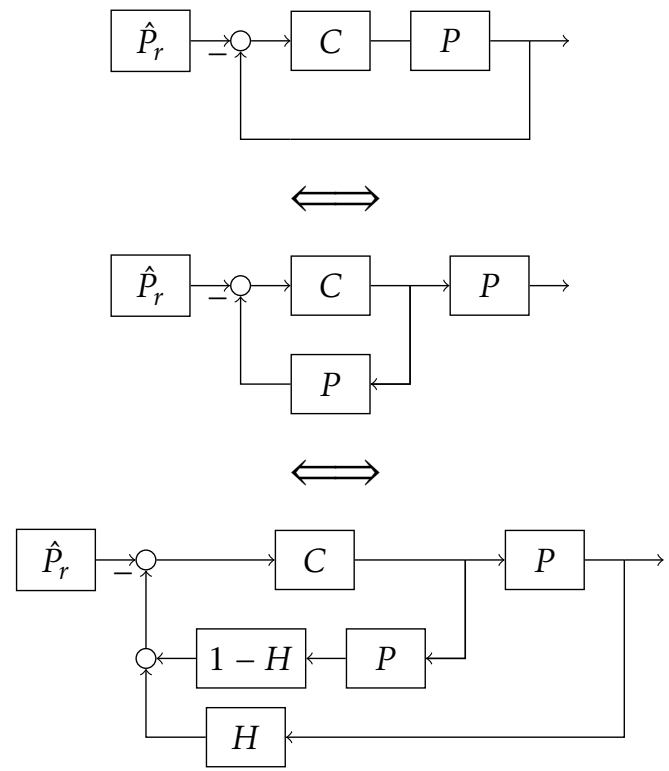

Figure 3.5: These reformulations illustrate how a regular control loop can be interpreted as inversion by means of feedback of a perfect model of the plant $P$, or as a mixture of both. Note that they do not require $P$ to have any particular properties, such as for example linearity.

of the IMC-parameter $Q$ as consisting of an approximate inverse $F$ and a filter $H$, that is

$$
Q=F H,
$$

where $H$ represents our trust in the measurements of the plant output compared to the output predicted by our model. If $H=1$ we only trust the measurements, and if $H=0$ we only trust the model.

\subsubsection{Complementary filter interpretation of error feedback}

One can use the complementary filter idea for another, perhaps less insightful, but nonetheless interesting, reinterpretation of a standard error feedback loop. The idea is to factorize the controller into an approximate inverse and a filter, similar to how $Q$ was interpreted in the previous section,

$$
C=H \hat{P}^{-1},
$$

and then use the filter part $H$ to reinterpret the error fed to the controller as the difference between the reference and the output of a complementary filter fusing the plant output with the reference. This is illustrated in Figure 3.7.

The intuition is that $H$ represents how much we trust the measured plant output compared to how strong our belief is that the plant will follow the reference 

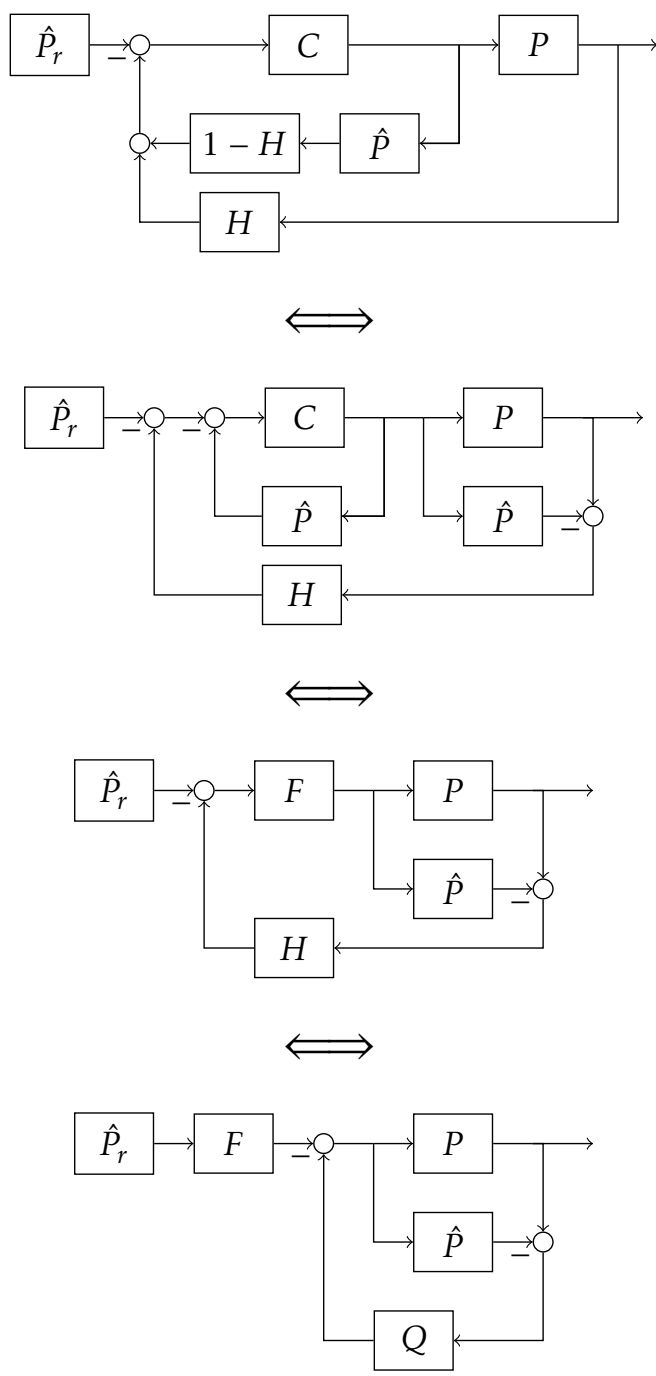

Figure 3.6: Starting from the concept of using a complementary filter to weight together feedback from the real plant $P$ and a model $\hat{P}$, one can arrive at the familiar IMC structure. The last step requires additivity from the approximate inverse $F$, which is present if $C$ and $\hat{P}$ are linear. 
without the intervention of feedback. The latter can be a reasonable belief for example in the presence of additional feedforward control. Since the reformulation in Figure 3.7 already contains an approximate inverse, adding feedforward requires little modification, as illustrated by Figure 3.8.

\subsubsection{Inversion of affine functions}

When control is described as being about inversion, the prime example is inversion of so called transfer functions, which are used to describe linear timeinvariant dynamical systems. For a linear transfer function, $P$, we have

$$
Y=P U,
$$

and inversion comes in the form of a multiplicative inverse, $P^{-1}$,

$$
P^{-1} Y=U,
$$

just like for any other linear function.

However, it is not often pointed out that when we model plants with exogenous disturbances, we mostly model them as affine functions,

$$
Y=P U+P_{d},
$$

as illustrated in Figure 3.9.

Likewise, when we talk about controlling a plant modeled by an affine transfer function, rarely do we explicitly mention inversion, opting instead to talk about canceling of the disturbance. It might be worthwhile to sometimes use the wording that inverting an affine function,

$$
P^{-1}\left(Y-P_{d}\right)=U,
$$

necessitates an additive inverse as well as a multiplicative one, as illustrated in Figure 3.10. This can serve as a good starting point for discussing how schemes with forward or inverse models, such as the one depicted in Figure 3.6, can be adapted to affine plants.

This observation was inspired by the block diagram transformation tables presented in the textbook by Oppelt [1964]. 


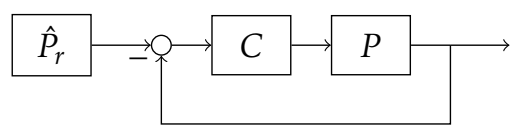

$$
\begin{gathered}
C=H \hat{P}^{-1} \\
\Longleftrightarrow
\end{gathered}
$$

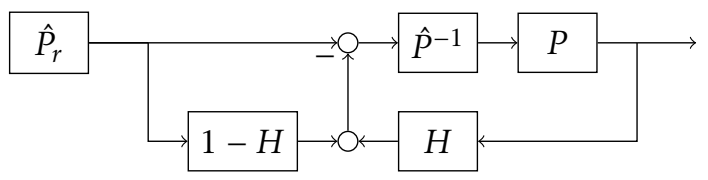

Figure 3.7: Error feedback from a directly measured output can be reinterpreted as feedback from the output of a complementary filter which fuses the reference and the measured output. This emphasizes the role of the controller as both a filter and as an inverse.

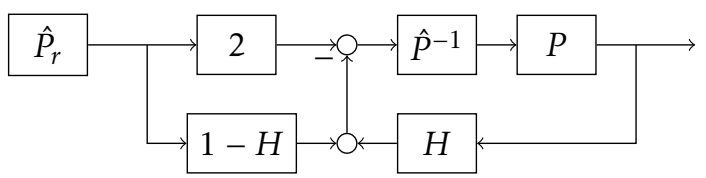

Figure 3.8: Adding feedforward to the scheme in Figure 3.7 is straighforward since an approximate inverse $\hat{P}^{-1}$ is already present. At frequencies where we trust the feedforward more than the feedback, $H$ should have a small amplitude.

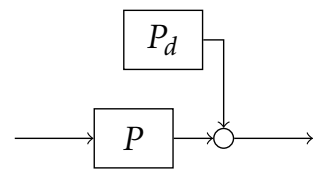

Figure 3.9: An affine transfer function.

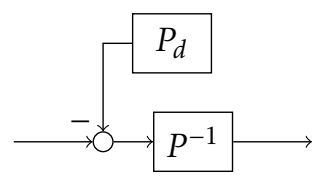

Figure 3.10: The inverse of an affine transfer function. 


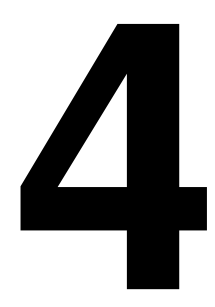

\section{From internal model principle to Youla parametrization}

This chapter introduces a line of reasoning that can be used as inspiration for teaching the Youla-Kučera parametrization (YKP), also known as Youla parametrization. The chapter is a somewhat revised and extended ${ }^{1}$ version of a paper by Hedberg et al. [2020] presented at the 21st IFAC World Congress.

\subsection{Introduction}

The Youla-Kučera parametrization of all stabilizing controllers for a given linear time-invariant system is an important result in control theory and often appears in courses on the subject. For stable plants, the YKP takes on a particularly simple form, and it is therefore often first introduced to students in that setting.

For unstable plants, the formulations become more involved and the transition from the stable case can be perceived as difficult to follow by some students. Especially the multivariable case, where the concept of coprime matrix factorization is an important tool, can pose difficulties.

The proposed line of reasoning, illustrated in Figure 4.1, takes as point of departure the internal model principle (IMP), which can be a useful conceptual tool for students when reasoning about control. Then two alternative controller structures, both using a model of the plant, are introduced; internal model control (IMC) and disturbance observer (DOB). These two structures are used to introduce a more general structure, based on factorization, which provides a natural bridge to the general formulation of the YKP.

\footnotetext{
${ }^{1}$ Most notably the addition of section 4.5.3, and some additions to sections 4.2.1, 4.4 and 4.5.2.
} 


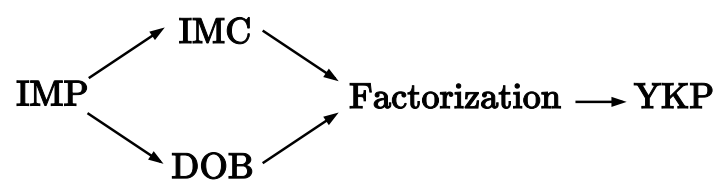

Figure 4.1: The internal model principle (IMP) is used to introduce internal model control (IMC) and disturbance observer (DOB). Comparing these control structures naturally leads to the concept of factorization, which is a bridge to the general Youla-Kučera parametrization (YKP).

\subsection{Preliminaries}

\subsubsection{The internal model principle}

The internal model principle (IMP) is the idea that in order to control a system and compensate for disturbances, the controller needs to have an understanding, i.e. an internal model, of how the system works and the nature of the disturbances. Intuitively this seems like a reasonable proposition, and it is in accordance with our everyday experience as humans.

The concept has been formalized for multivariable linear systems by Francis and Wonham [1975], and generalized to a setting of abstract automata by Wonham [1976].

An interesting paper by Conant and Ashby [1970] derives a general principle using a broad concept of sets and mappings together with an argument based on entropy. The title of that paper, Every good regulator of a system must be a model of that system, succinctly summarizes the internal model principle even though the paper does not refer to it by that name. References to this paper seem to be rare in control literature.

The internal model principle does not seem to figure prominently in textbooks on automatic control. Åström and Murray [2008] for example mentions it in passing when discussing observer-based state feedback, and Corriou [2004] calls it "a recommendation of general interest", briefly gives a frequency description and references the work of Francis and Wonham [1975].

Of the surveyed textbooks, Goodwin et al. [2001] discusses the IMP the most. There, the internal model principle is discussed in relation to disturbance rejection as well as reference tracking. It is brought up both for single input, single output (SISO) systems in transfer function form and for multiple input, multiple output (MIMO) systems in state space form. In the latter case its relation to disturbance estimation is discussed. It is also mentioned in relation to achieving integral action in LQ control.

In some literature, e.g. Åström and Wittenmark [1984], Maciejowski [1989], Chen [1999], Franklin et al. [2002], Dorf and Bishop [2008], the IMP is only used 
to refer to the principle that in order to compensate for a persistent disturbance, the controller needs to contain a model of the system generating the disturbance.

\subsubsection{Internal model control}

Internal model control (IMC) as introduced in Garcia and Morari [1982] refers to a particular control structure that uses an "internal model to predict the effect of the manipulated variables on the output". The book by Morari and Zafiriou [1989] is a widely cited reference, in which IMC is the basis for a robust control design procedure.

Brosilow and Tong [1978] introduces the same concept, under the name of inferential control, and focuses on the estimation aspect, making the connection between the IMC and DOB ideas clear.

The main idea is that only the deviation from the predicted output, which can be interpreteted as an output disturbance estimate $\hat{d}_{y}$, is fed back to the controller.

IMC is commonly presented with a block-diagram like the one in Figure 4.2, although García et al. [1989] ${ }^{2}$ observes that the two-degree-of-freedom (2-DOF) structure presented in Figure 4.3 is preferable. They also give a brief historical summary of how the concepts leading up to IMC control developed.

Horowitz [1963] discusses how 2-DOF structures are all equivalent, and among the examples we find the IMC structure under the name of model feedback (chapter 6, figure 6.1-1f). The book also offers the following illuminating quote on the equivalence of 2-DOF controllers:

"... it destroys the mystique of structure which seems to some to be of great importance in feedback theory. The designer need not fear that, if he were only clever enough, he could find some exotic structure with new and wonderful properties."

Frank [1974] gives a good account of how the ideas of model feedback evolved, and also provides an experimental design procedure (in section 2.7.1) that nicely illustrates the relation between IMC and modeling.

There is a one-to-one correspondence between an error feedback controller $C$, shown in Figure 4.4, and an IMC controller $Q$ such as in Figure 4.2, given by

$$
C=\frac{Q}{1-\hat{P} Q}, Q=\frac{1}{C+\hat{P} C} .
$$

In case of a perfect model $\hat{P}=P$, the transfer functions characterizing the closed loop system (the so-called Gang of Four, see e.g. Åström and Murray [2008])

\footnotetext{
${ }^{2}$ The title of that paper includes model predictive control, a term that has taken on a slightly different meaning in modern control terminology. The process control oriented textbook Marlin [2000] uses it in the same sense as García et al. [1989] for IMC-like control.
} 


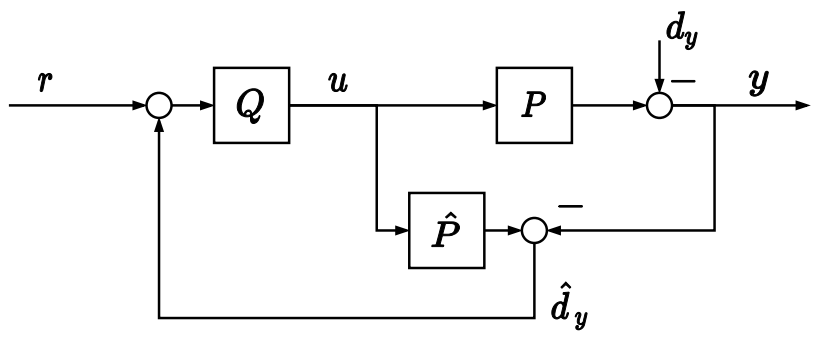

Figure 4.2: IMC is often introduced using a 1-degree-of-freedom (1-DOF) structure.

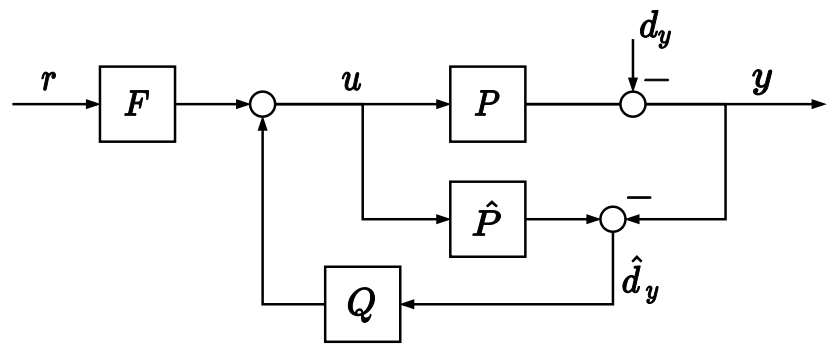

Figure 4.3: García et al. [1989] advocates using a 2-DOF IMC structure. This structure illustrates the interpretation of error feedback as feedforward combined with disturbance estimation and rejection.

takes on a particularly simple form,

$$
\left[\begin{array}{l}
y \\
u
\end{array}\right]=\left[\begin{array}{cc}
P Q & P(1-P Q) \\
Q & 1-P Q
\end{array}\right]\left[\begin{array}{c}
r \\
d_{u}
\end{array}\right],
$$

making them easy to analyze.

\subsubsection{Disturbance observer}

Another, less widespread, controller structure that also uses an internal model is the disturbance observer structure (DOB), where an inverse model of the system is used to estimate an input disturbance $d_{u}$ and try to cancel that disturbance. The concept is illustrated in Figure 4.5, where the filter $H$ should ideally be equal to 1 , but has to have sufficient relative degree to ensure realizablility.

The term disturbance observer is introduced in Nakao et al. [1987], and Oboe [2018] gives a contemporary (and enthusiastic) introduction. 


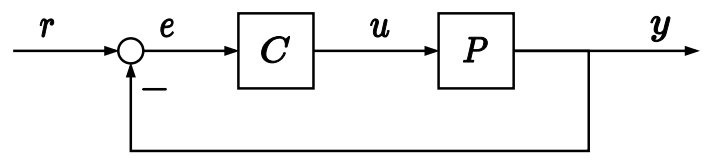

Figure 4.4: A common 1-DOF feedback controller structure.

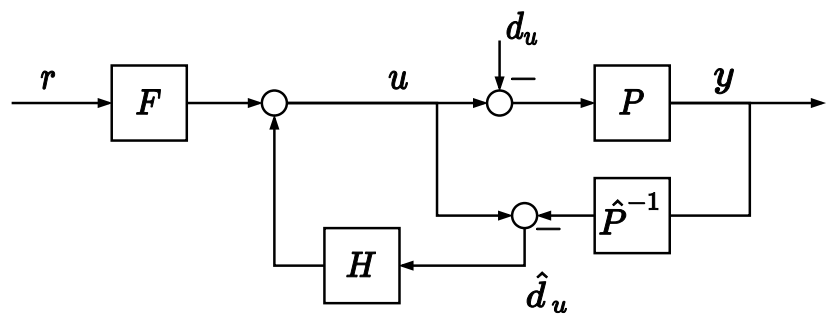

Figure 4.5: The disturbance observer controller structure uses the model to estimate an input disturbance $d_{u}$, instead of an output disturbance $d_{y}$ like IMC does.

\subsubsection{The Youla-Kučera parametrization}

The idea behind the Youla-Kučera parametrization (YKP) was presented independently in papers by Youla et al. [1976] and Kučera [1975]. It can be seen as a development of the idea behind internal model control.

The YKP describes the set of all controllers that stabilize a given linear system, and parametrizes that set by a stable transfer function, often denoted $Q$. The fact that the closed loop transfer functions become linear in $Q$ makes the YKP a powerful tool for optimization-based synthesis of controllers.

\subsection{Proposed procedure}

In this section we present a suggestion for how the previously discussed topics can be introduced to students.

\subsubsection{Introduce the internal model principle}

Introduce the idea that to control something, the controller needs knowledge about the process to be controlled. Appeal to everyday experience to show that this proposition is reasonable.

Mention that this intuitive principle can be formalized in different theoretical 


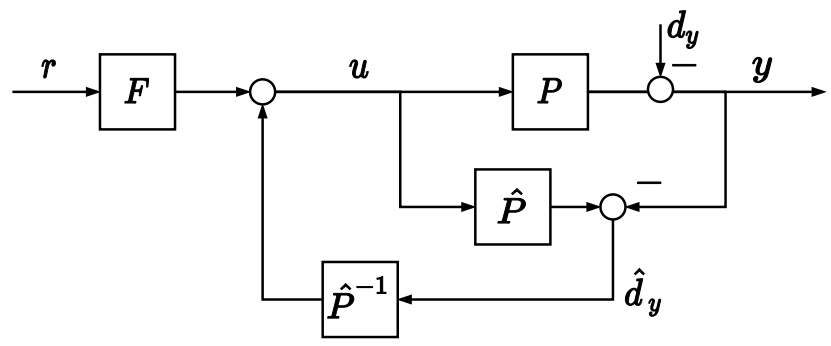

Figure 4.6: Ideal IMC, where the estimated output disturbance is fed through an inverse of the model.

settings, one of which is linear time-invariant systems.

Note that in our field, system knowledge often takes the form of a mathematical model. Add that the power of feedback control is that often a simple approximation can suffice as model, in some cases as simple as knowing the sign of the steady state gain.

\subsubsection{Introduce IMC and DOB}

Explain that one way of using a model in a controller, is to compare the actual system behaviour with the behaviour predicted by the model, and then using only the difference, i.e. the unexpected behaviour, to make an adjustment.

Introduce the IMC structure in Figure 4.3 as an example of the above, and mention that here all unexpected behaviour is in a sense interpreted as an output disturbance $d_{y}$. Note that ideally, we would like to use a perfect model inverse, as in Figure 4.6, but that this is rarely possible.

Now, mention that it is also possible to regard all unexpected behaviour as an input disturbance, and introduce the DOB structure in Figure 4.5 as an example. Note that we would ideally like to achieve the structure in Figure 4.7, but that this, again, is generally not possible.

\subsubsection{Demonstrate equivalence between IMC and DOB}

Note that the structure in Figure 4.5 can easily be turned into Figure 4.3 by first "sliding" the inverse model $\hat{P}^{-1}$ down through the summation junction and then selecting $H=Q \hat{P}$.

\subsubsection{Interpret in terms of factorization}

Introduce Figure 4.8 as a way of describing both structures, and note that when changing between IMC and $\mathrm{DOB}$, one invariant is that $N M^{-1}=\hat{P}$, i.e. that $N$ 


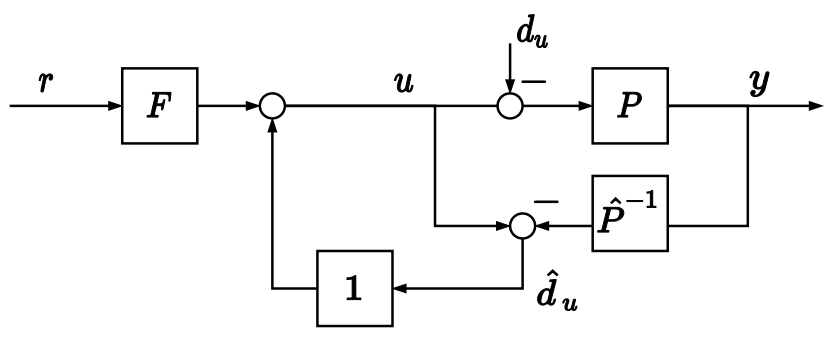

Figure 4.7: Ideal DOB, where the estimated input disturbance is cancelled directly.

and $M^{-1}$ is a factorization of $\hat{P}$.

Remark that this might lead the curious mind to wonder if other interesting controller structures might be obtained by using different factorizations.

\subsubsection{Introduce the polynomial factorization}

Note that since we are dealing with a rational transfer function $\hat{P}=\frac{\hat{B}}{\hat{A}}$, one natural choice to investigate would be to simply let $N=\hat{B}$ and $M=\hat{A}$. Remark that this in fact gives us two stable transfer functions in the structure in Figure 4.9, since the polynomials by definition have no poles.

Admit that of course, polynomials are not proper transfer functions, and if we would like to use the structure in Figure 4.8 for implementation we can instead choose a factorization

$$
N=\hat{B} C^{-1}, M=\hat{A} C^{-1}
$$

where $C$ is a polynomial without roots in the right half plane and of sufficient degree to make both $N$ and $M$ proper and thus realizable. Illustrate this by Figure 4.10 .

If suitable, show Table 4.1 to illustrate how a given IMC controller translates to

Table 4.1: Parameter choices to achieve equivalent controllers in different formulations.

\begin{tabular}{lccc}
\hline Structure & $Q_{v}$ & $N$ & $M$ \\
\hline IMC & $Q$ & $\hat{P}$ & 1 \\
DOB & $Q \hat{P}$ & 1 & $\hat{P}^{-1}$ \\
PF & $Q \hat{A}^{-1}$ & $\hat{B}$ & $\hat{A}$ \\
IPF & $Q C \hat{A}^{-1}$ & $\hat{B} C^{-1}$ & $\hat{A} C^{-1}$ \\
\hline
\end{tabular}




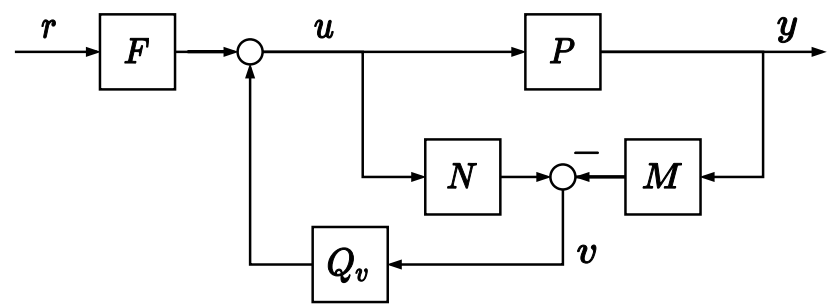

Figure 4.8: A general 2-DOF IMC-like controller structure, similar to what Chen [1999] calls the controller-estimator or plant-input-output-feedback structure.

the DOB, the polynomial factorization (PF) and the implementable PF (IPF).

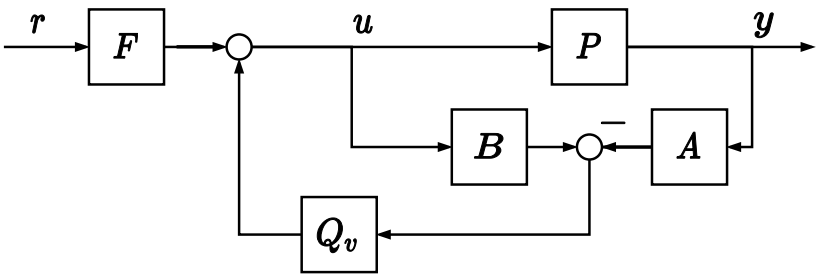

Figure 4.9: For a rational SISO system, factorization by the numerator and denominator polynomials yields stable transfer functions.

\subsubsection{Introduce the concept of YKP using IMC}

Signal a change in perspective by clarifying that the previous discussion was about choosing controller structures based on the intuition of the IMP, but that we will now use the same structures to describe the set of all linear controllers that stabilize a given system. To do this we consider the case when the model is perfect, $\hat{P}=P$.

Derive, or simply introduce, the transfer functions (4.2) characterizing the closed loop system when using an IMC controller with a perfect model. Note that if $P$ is stable, the "Gang of Four" (4.2) will be stable for any stable $Q$.

Note, or prove (e.g. Morari and Zafiriou [1989]), that not only does every stable $Q$ give a stable closed loop system for a stable $P$, but every stabilizing feedback controller $C$ can be obtained by a stable $Q$ and the expression (4.1). 


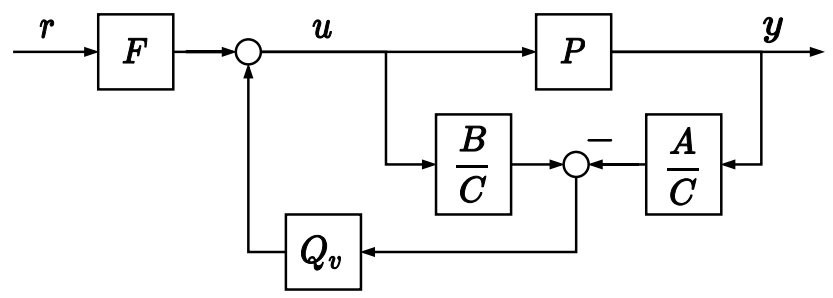

Figure 4.10: To keep the factorized structure in implementation, a filter polynomial $C$ can be introduced to ensure properness, without restricting design choices.

\subsubsection{Extend to the unstable case}

Return to the transfer functions (4.2) and note that if $P$ is unstable, it is sufficient to find a stable $Q$ that stabilizes the critical transfer functions

$$
P Q, P(1-P Q)
$$

to achieve internal stability for the closed loop system. Remark that we would like a parametrization without such constraints on the parameter $Q$.

Demonstrate how this can be achieved by introducing

$$
Q=Q_{0}+A^{2} Q_{1} \text {. }
$$

And writing the critical transfer functions (4.4) as

$$
P Q_{0}+A B Q_{1}, P\left(1-P Q_{0}\right)-B^{2} Q_{1} \text {. }
$$

Note that if $Q_{0}$ is chosen to stabilize (4.4), we are free to choose any stable $Q_{1}$, since $B$ and $A$ are stable by definition. Compare to homogeneous and particular solutions of a differential equation.

Note, or prove (e.g. Morari and Zafiriou [1989]), that given a stabilizing $Q_{0}$ all stabilizing controllers are then parametrized by the choice of a stable $Q_{1}$.

\subsubsection{Block-diagram representations and interpretations}

Choose Q in the IMC controller as (4.5) and show, by inserting into Figure 4.9, that the corresponding controller can be described as in Figure 4.11, illustrating that the YKP can be interpreted as two IMC-loops.

Now remark, or if suitable show, that the block diagram in Figure 4.11 can by some deft manipulation be turned into the one depicted in Figure 4.12, thus illustrating another interpretation of the YKP as first applying an IMC-loop for disturbance rejection and then applying an outer, stabilizing, feedback loop.

Since Figure 4.12 does not seem to be common in textbooks a few remarks are in 


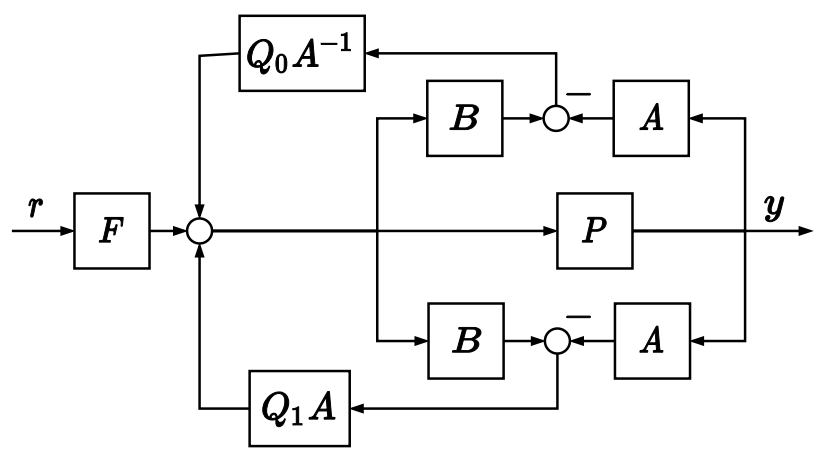

Figure 4.11: The general YKP can be interpreted as two IMC-loops, where one is freely parametrized.

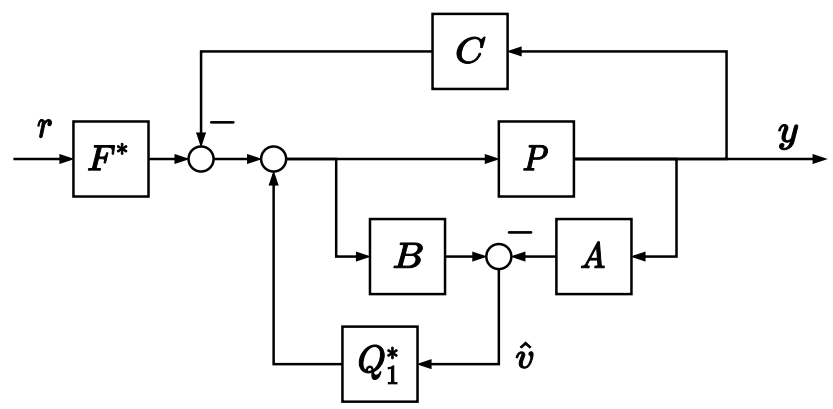

Figure 4.12: An alternative interpretation of the YKP is as an inner disturbance rejection, or "model following", loop combined with an outer stabilizing loop.

order. $C$ is the feedback controller obtained from inserting $Q_{0}$ into (4.1), and $Q_{1}^{*}$ and $F^{*}$ can be chosen as stable transfer functions of sufficient relative degree, but are not the same as $Q_{1}$ and $F$ in Figure 4.11. In deriving Figure 4.12 it is helpful to observe that the stability of (4.6) implies that $Q_{0}$ can always be factorized as $Q_{0}=Q_{0}^{*} A$ where $Q_{0}^{*}$ is stable, and that $\left(1-Q_{0} P\right)$ can be factorized in a similar manner.

\subsection{Pedagogical merits}

The concepts introduced above are standard fare in control theory, and we do not pretend that our presentation of the material contains any novel interpretations. In a brief survey of textbooks we have however not found the same way of linking the concepts together. 
While we have not had the opportunity of trying this procedure in teaching, we would like to highlight what we think are the pedagogical strengths:

a) We believe that exposing students to the idea of the IMP early, and stressing its generality, can aid them in developing a solid understanding of the close connection between modelling, estimation and control. The core of the IMP is quite intuitive, and so the cost of including it in a course should be relatively low compared to the potential benefit.

b) Introducing the DOB structure alongside IMC can be a good opportunity to illustrate how block diagram manipulations reveal different interpretations of the same controller. It also illustrates how control can be regarded as estimation and compensation of disturbances.

c) When treating the YKP, it might be beneficial for student understanding to minimize the differences between the block diagrams used to illustrate the stable and the unstable case respectively. In this regard Figure 4.9 and Figure 4.12 could be advantageous compared to using Figure 4.2 together with e.g. Figure 4.13 or Figure 4.14.

d) Introducing factorization as a natural tool in the SISO case will likely facilitate an eventual transition to the use of coprime (transfer) matrix factorization for the MIMO case.

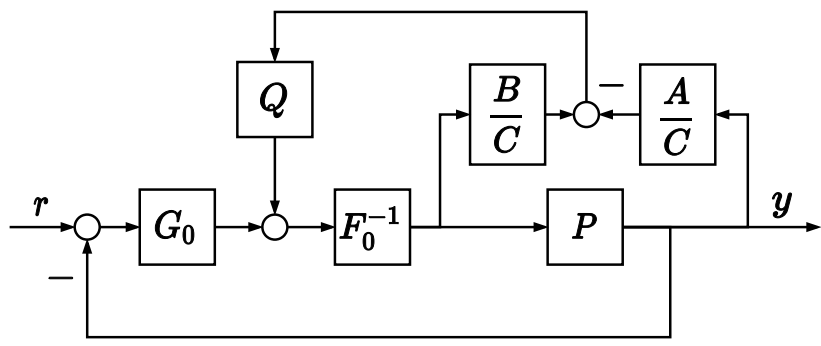

Figure 4.13: Åström and Murray [2008] uses a similar block-diagram to illustrate the general YKP. The transfer functions $G_{0}$ and $F_{0}^{-1}$ are obtained from a coprime factorization of a stabilizing controller. $C$ is a polynomial with roots in the $L H P$.

\subsection{Possible extensions}

Finally we would like to note some illustrative examples that could, depending on the type of course, fit well together with the proposed procedure. 


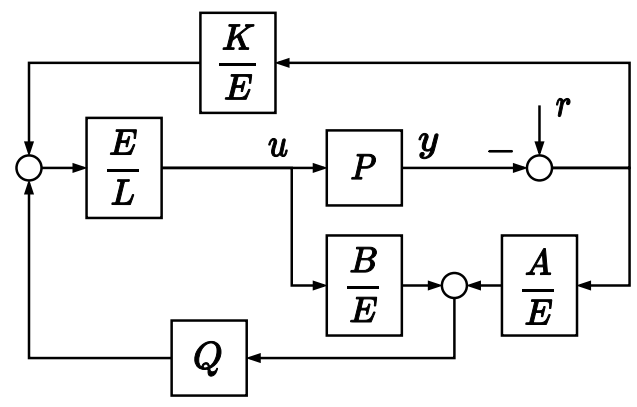

Figure 4.14: Goodwin et al. [2001] illustrates the general YKP using this structure. The polynomials $K$ and $L$ constitute a polynomial factorization of a stabilizing controller, and the roots of the polynomial $E$ is part of the closed-loop poles. Note that E can be eliminated from the block-diagram.

\subsubsection{Derive the PID controller from DOB}

Using the DOB structure in Figure 4.2 with a second order model,

$$
\hat{P}=\frac{1}{s^{2}+a s+b},
$$

and a first order low-pass filter,

$$
H=\frac{1}{1+s T}
$$

it is straightforward to derive the equivalent error feedback controller,

$$
C=\frac{s^{2}+a s+b}{s T}=\frac{s}{T}+\frac{a}{T}+\frac{b}{T s} \triangleq K_{p}\left(1+\frac{1}{T_{i} s}+T_{d} s\right),
$$

from the structure in Figure 4.15. The result is a PID controller where the gain $K_{p}$ is inversely proportional to the time constant $T$ of the filter $H$.

This example can serve as a good opportunity to elaborate on the fact that a simple model can often be enough to achieve acceptable control performance, and that this is one reason for the prevalence of PID control.

In the same spirit one can also mention that a first order low-pass filter is often a good place to start when investigating a signal processing problem. ${ }^{3}$

\subsubsection{Discuss cascade control}

The notion that IMC and DOB contain estimations of output/input disturbances can be taken further by noting that every factorization $N$ and $M^{-1}$ of the system corresponds to viewing the system as composed of two subsystems in series, and

\footnotetext{
${ }^{3}$ To quote Glad and Ljung [2000]: The basic principle, as in all engineering work, is "to try simple things first".
} 


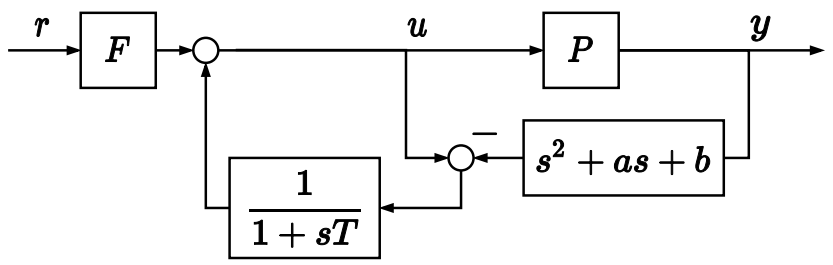

Figure 4.15: A PID controller in disguise.

to interpreting the signal $v$ in Figure 4.8 as the estimate of a disturbance entering between these two subsystems.

If the system is indeed composed of two physical subsystems in series, and we have access to a measurement of the intermediate signal, the IMC/DOB framework can easily incorporate this measurement, giving rise to a cascaded IMC control structure, as illustrated in Figure 4.16. Further discussions of IMC in cascade structures can be found in Semino and Brambilla [1996], however with a focus on parallel structures. Cesca and Marchetti [2005] examines tuning of series cascade IMC. A remark about series cascade IMC can also be found in the process control textbook Bequette [2003] (section 10.5).

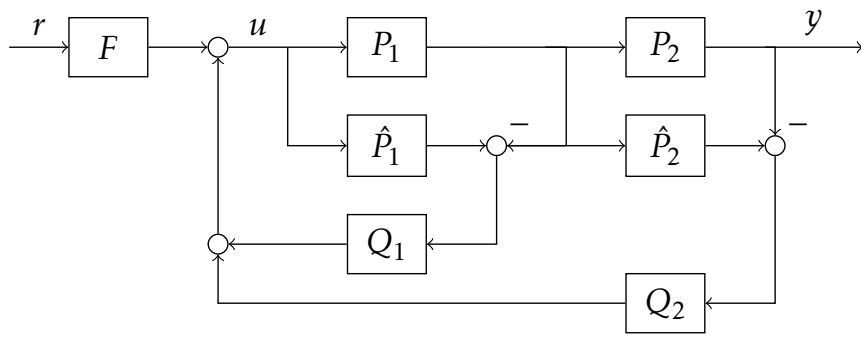

Figure 4.16: IMC applied in a cascade structure.

\subsubsection{Relate feedforward and IMC}

While traditional discussions of feedback control might not emphasize the need for models, discussions of feedforward control naturally concerns models and their inversion, and are therefore a good opportunity to also talk about internal model control.

A common scheme for feedforward, illustrated at the top of Figure 4.17, is to use a plant model $\hat{P}$ to predict the effect of the feedforward signal, and use this as a reference for the feedback controller. This idea is closely related to the ideas behind IMC, and that relation can be nicely demonstrated using the block diagram manipulations illustrated in Figure 4.17. 

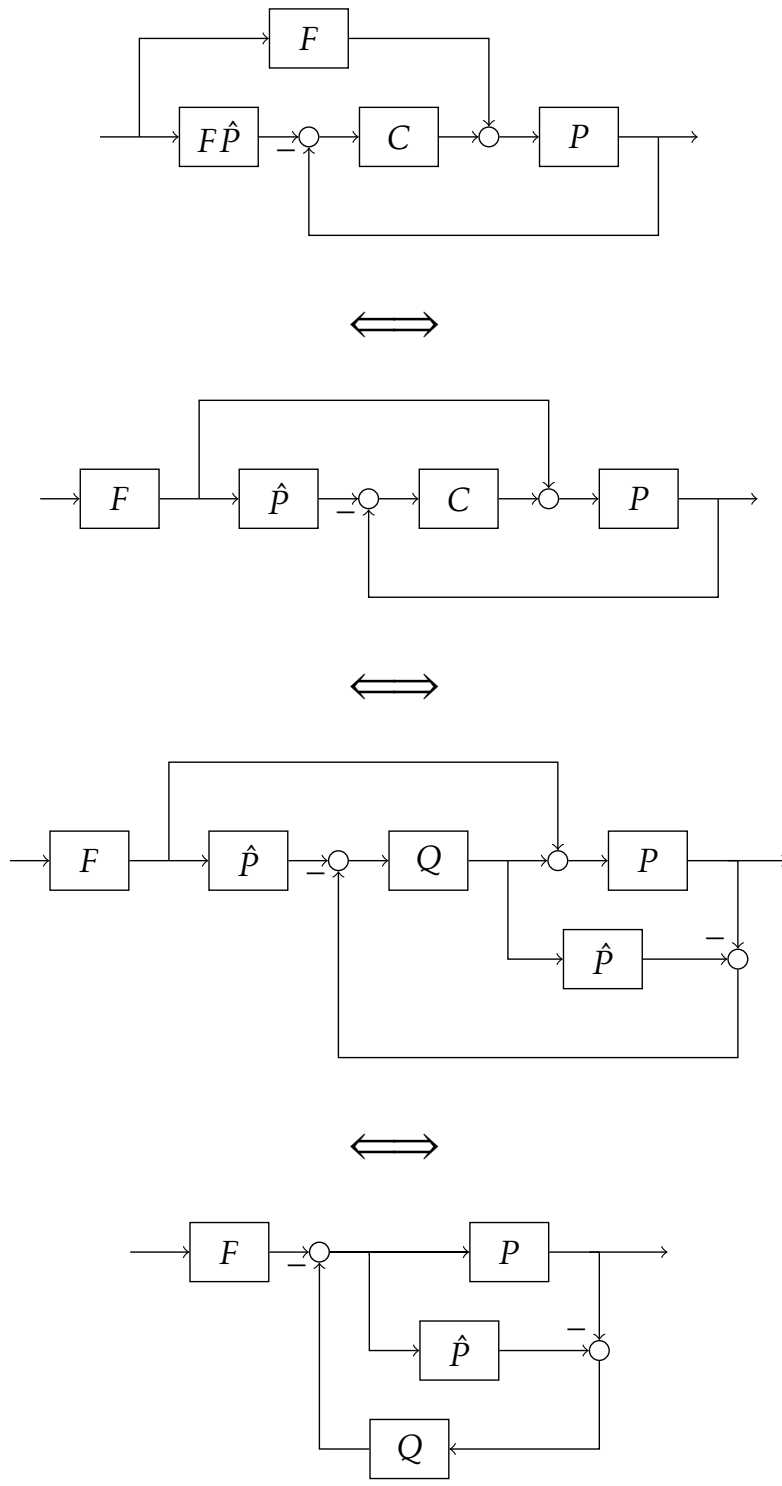

Figure 4.17: A traditional feedforward scheme can be transformed into a two degree of freedom IMC scheme by rewriting the feedback controller $C$ in its IMC form and performing some block diagram manipulation. Since $F$ is often chosen to approximate $\hat{P}^{-1}$ well, at least over the pass band of $C$, it is common to see the block $F \hat{P}$ replaced by unity in the topmost diagram. 


\section{Case study: Tool position estimation using inertial measurements}

This chapter is a slightly revised version of the paper Hedberg et al. [2017], which was presented at the 20th IFAC World Congress. It compares two filtering approaches for using inertial measurements to improve on purely kinematic position estimates.

\subsection{Introduction}

Industrial robot tool position control relies heavily on model-based feedforward, with a feedback loop based on motor angle measurements. When models are not accurate enough additional measurements can be used to improve the accuracy of the tool position estimate. One possibility is to use inertial measurements, i.e. acceleration and angular velocity, of the tool. This work experimentally investigates how an Intertial Measurement Unit (IMU) mounted on the robot tool can improve the estimates obtained from forward kinematics based on motor angles.

Position estimation based on inertial measurements is a well studied problem, and in this light, the contributions of this work are:

- The application to a 6-DOF industrial robot using highly accurate reference sensors, giving a qualitative feel for possible performance.

- Investigating the complementary filter (CF) for this kind of application, finding that it performs similarly to the more well-known Extended Kalman filter $(\mathrm{EKF})$ and analysing the reasons behind this. 

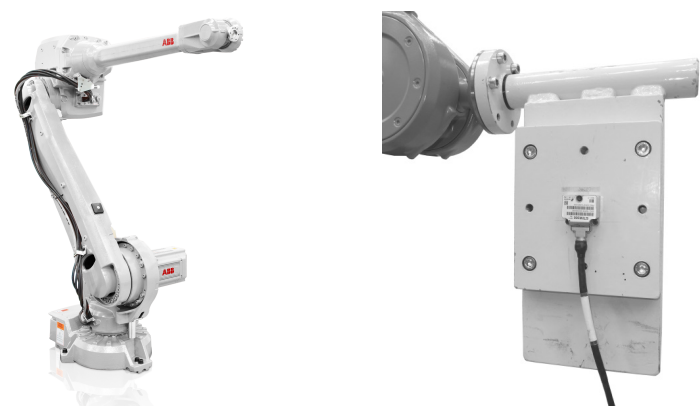

Figure 5.1: To the left an ABB IRB4600 Industrial Robot and to the right a Sensonor STIM300 IMU and test weight mounted on the robot.

\subsection{Related work}

For a general introduction to IMU-based estimation see for example Hol [2011]. In the area of industrial robots, similar work has been carried out by Olofsson et al. [2016] and Chen and Tomizuka [2014]. The former is similar to the present work in scope; an EKF was compared to a Particle filter (PF) instead of a CF. In the latter, a flexible-joint model of the robot is used, and joint-angles are estimated using individual KF:s.

The idea behind the CF was first introduced in a patent, Wirkler [1951], and the name was, to the authors' best knowledge, introduced in Anderson and Fritze [1953]. The method has been well studied in the context of IMU-based estimation, especially attitude estimation. An introduction to the CF in that context is given in Jensen et al. [2013]. For an illuminating discussion of the relation between complementary filtering and Kalman filtering see Brown [1972], also in the context of intertial navigation. Regarding robotics there are examples of CF applications in Roan et al. [2012], where it was applied to joint angle estimation on a humanoid robot, and Axehill et al. [2014], where it was applied to a parallel kinematic robot.

\subsection{System overview}

The experimental setup used in this work consists of an industrial robot with motor angle measurements, an IMU mounted on the robot tool and a high-precision tracking system for reference measurements. A more detailed presentation of the setup is given by Norén [2014].

\subsubsection{Industrial robot}

The industrial robot used in the experiments is an IRB4600, shown in Figure 5.1. The robot is rated for a $45 \mathrm{~kg}$ payload, weighs around $420 \mathrm{~kg}$ and is roughly 2.4 meters in upright position, see ABB [2016] for details. 
The robot is modelled using kinematics only, dynamic effects like friction or flexibilities are not modelled.

\subsubsection{Inertial measurement unit}

The sensor for measuring acceleration and angular velocity is a STIM300 IMU, which can be considered a high-end IMU, see Sensonor AS [2016] for specifications. In Figure 5.1 the sensor is shown mounted on the robot. To be able to use the sensor measurements and relate the measured acceleration and angular velocity to the robot motion it is necessary to estimate the position and orientation of the sensor relative to the robot. The first step is to compute the internal parameters of the sensor, scaling and offset, and the orientation of the sensor with respect to the robot. In the second step the position of the sensor is estimated by moving the robot. For details see Norén [2014].

\subsubsection{Sensor for evaluation purposes}

An LTD840 laser tracker system from Leica Geosystems was used to obtain accurate position measurements for use as a reference. The system has an accuracy on the order of $0.1 \mathrm{~mm}$ in the conditions under consideration, see Leica Geosystems [2016], and measurements are provided at $1 \mathrm{kHz}$ sample rate. A limitation of this system is that it does not provide orientation measurements.

\subsection{Estimation methods}

\subsubsection{Complementary filter}

Complementary filtering is a way of approaching the problem of fusing measurements/estimates with different noise characteristics.

The idea of the CF is that in the absence of measurement noise the filter should be a perfect estimator. That is, there should be no dynamics between the true signal and the estimate, as those dynamics would distort the estimate even with perfect measurements. This is referred to as the complementary constraint. Other terms found in literature are distortionless filtering and exact dynamic filtering, see Brown and Hwang [1997]. It can also be viewed as a min-max approach, see Brown [1972].

In the case where nothing is known about the statistical properties of the true signal, i.e. the process noise completely dominates the measurement noise, any optimal estimate will satisfy the complementary constraint.

As such, a complementary filter is equivalent, as is any linear filter, to a Kalman filter derived under certain assumptions on the system. The principle behind the complementary filter is essentially the same as behind the so called error-state or indirect Kalman filter, see Brown [1972] and Maybeck [1979]. 
Having, by the choice of complementary filtering, assumed that the underlying signal is unpredictable the only remaining design parameters are the assumptions on the relative spectral power densities of the measurement noise signals.

Let $\hat{p}_{\mathrm{FK}}$ be the forward kinematics position estimate and $\hat{p}_{\mathrm{IMU}}$ the position estimate obtained from integrating the IMU signals:

$$
\begin{aligned}
\hat{p}_{\text {FK }} & =p+v \\
\hat{p}_{\text {IMU }} & =p+w
\end{aligned}
$$

Here $p$ is the true position and $v$ and $w$ are noise terms representing the errors in the estimates. Then, in order to satisfy the complementary constraint, the final estimate has to be of the following form, given in transfer function notation:

$$
\begin{aligned}
\hat{P}(s) & =G(s) \hat{P}_{\mathrm{FK}}(s)+(1-G(s)) \hat{P}_{\mathrm{IMU}}(s) \\
& =P(s)+G(s) V(s)+(1-G(s)) W(s)
\end{aligned}
$$

Thus we see in equation (5.2) that the filter $G(s)$ only affects the error terms, and we want to choose $G$ as to minimize the contribution of the error terms $V(s)$ and $W(s)$ to $\hat{P}(s)$.

When the noise terms have a low- and high-frequency character respectively, the tuning process reduces to choosing a cut-off frequency for the filter $G(s)$ and an appropriate roll-off. Here, the low-frequency information is in the forward kinematics as that estimate has no temporal drift, but fails to account for dynamic effects such as link flexibility and friction. The IMU-measurements on the other hand have a low-frequency bias drift, but captures rapid changes well.

Implementation and tuning of the complementary filter was done manually in a matter of hours, and resulted in choosing $G(s)$ as a second-order low-pass filter with a cut-off frequency of slightly less than $30 \mathrm{~Hz}$.

The estimated states were tool position in world coordinate frame and tool orientation as a quaternion. The filtering was performed in two steps, first the orientation estimate was updated and this update was then used to align the acceleration measurements with the world coordinate frame before integrating and updating the position estimate.

For simplicity, the same filter was used for all estimated states. A natural extension would be to tune different filters for the position and orientation estimates.

\subsubsection{Extended Kalman filter}

The Kalman filter (KF) and its extension to non-linear systems in the form of the Extended Kalman filter (EKF) are well-known methods, see for example Gustafsson [2012] for details. 
The Kalman filter is an optimal solution to the linear filtering problem with Gaussian noise, in the sense that its estimates are unbiased with minimum variance. The Kalman filter approach relies on models of the system dynamics and has a large number of design parameters compared to the complementary filter.

In the EKF, the algorithm used is basically the same as in the KF, but the nonlinear equations are linearized around the current state estimate at each timestep. In the non-linear case the optimality of the KF is lost, but given "nice" non-linearities it is reasonable to expect the EKF to perform well.

Here, robot tool position is modelled by a constant acceleration model and tool orientation by a constant angular velocity model. The IMU is further modelled as having a separate bias for each axis and type of signal, giving the states of the EKF as position, velocity and acceleration of the tool in the world reference frame, orientation as a quaternion, angular velocity in the sensor frame and finally sensor biases:

$$
x=\left[p, v, a, \mathfrak{q}, \omega, b_{a c c}, b_{g y r}\right]
$$

Time update equations are straightforward given the constant-acceleration and constant-velocity assumptions, see for example Gustafsson [2012]. The measurement equations can be written, with scaling factors from calibration omitted for simplicity, as:

$$
\begin{aligned}
y_{p} & =p+e \\
y_{\mathfrak{q}} & =\mathfrak{q}+e \\
y_{a c c} & =R_{B}^{I}(a+g)+b_{a c c}+e \\
y_{g y r} & =\omega+b_{g y r}+e
\end{aligned}
$$

Where $y_{p}$ and $y_{q}$ are the forward kinematic estimates, $y_{a c c}$ and $y_{g y r}$ the IMUmeasurements, $e$ represents measurement noise, $R_{B}^{I}$ a rotation from the world frame to the IMU-frame and $g$ gravity.

The EKF was tuned using a genetic algorithm for global optimization, which was left to run for a total period of several days. This resulted in a model with estimated process noise ten orders of magnitude larger than the estimated measurement noise.

\subsection{Trajectories}

Two trajectories were used for experimental evaluation. The first, shown in Figure 5.2, followed a benchmark path for industrial robots, part of the ISO 9283:1998 standard. It has previously been presented by Moberg [2010]. For this trajectory, the target tool speed was $200 \mathrm{~mm} / \mathrm{s}$. 


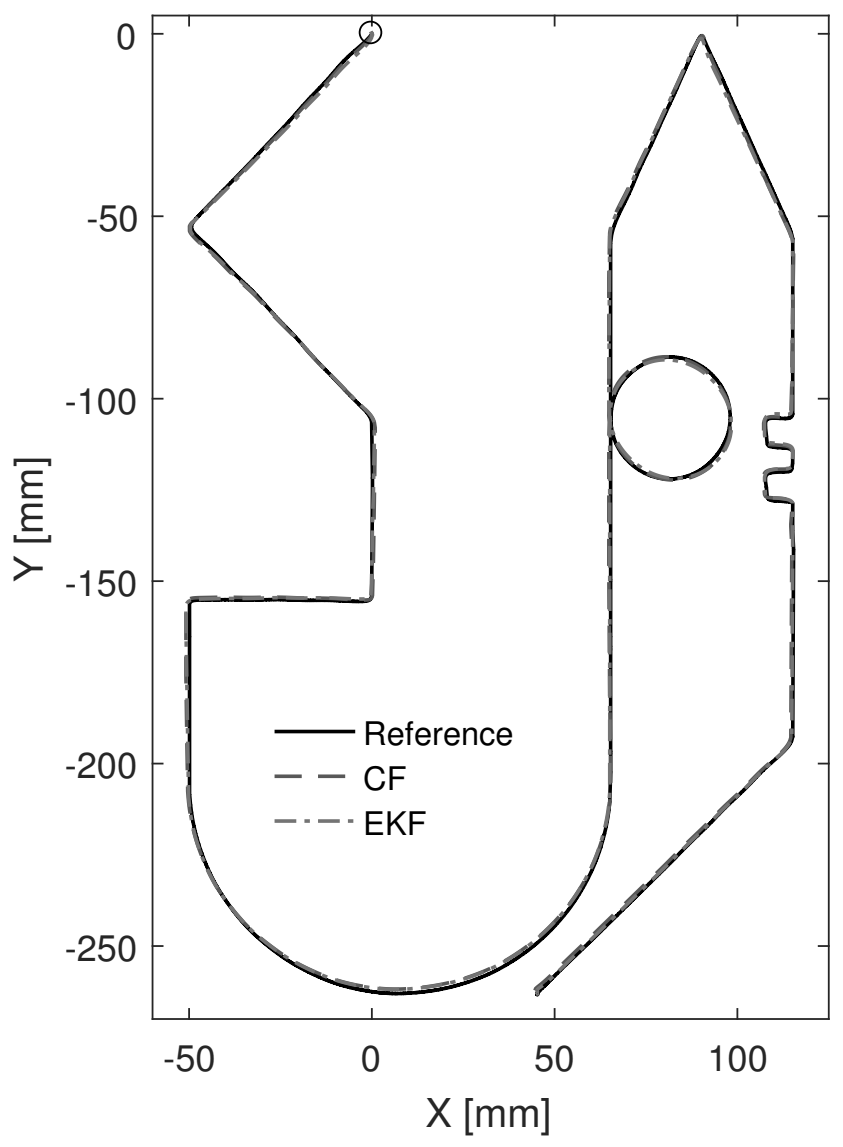

Figure 5.2: Estimates from the ISO trajectory. The trajectory is chosen to be representative of challenging industrial applications. The starting position is marked with a circle, and the target tool speed was $200 \mathrm{~mm} / \mathrm{s}$. 


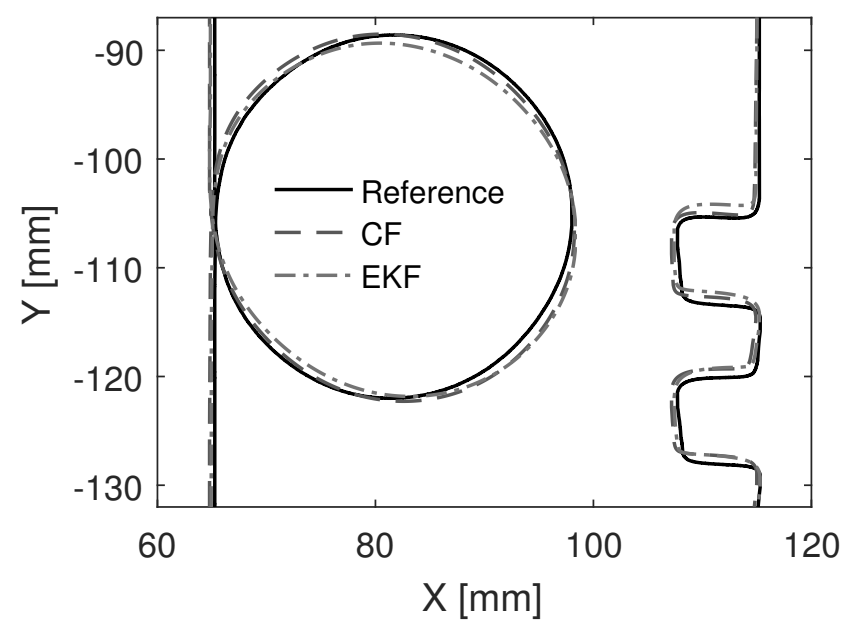

Figure 5.3: Details from the ISO-trajectory. Note that the travelled direction in the right part of the figure is downwards.

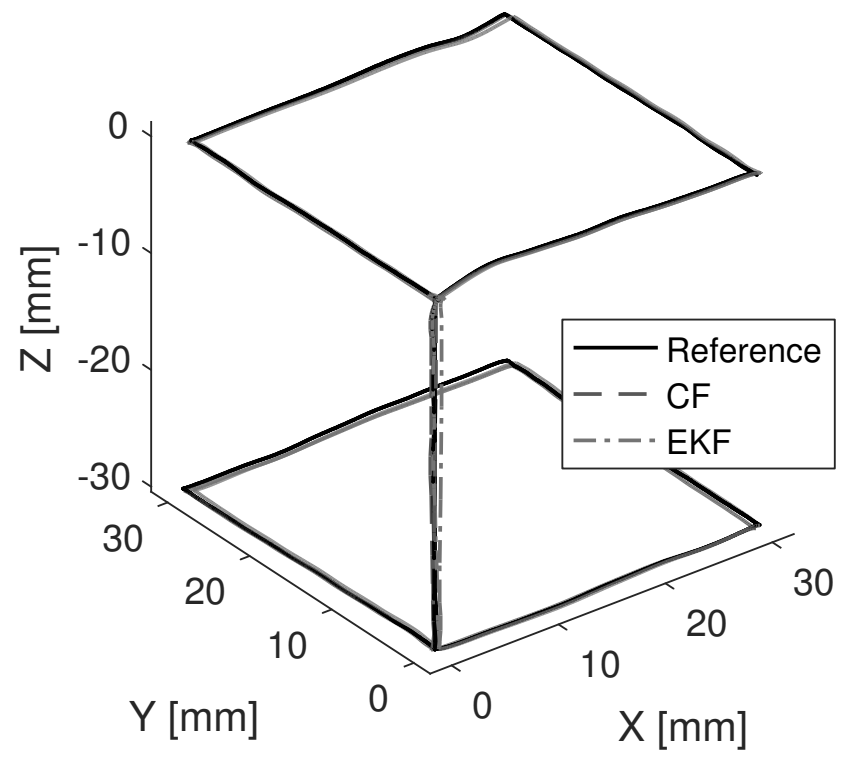

Figure 5.4: Estimates from the cube trajectory, using a target tool speed of $80 \mathrm{~mm} / \mathrm{s}$ and high precision mode, i.e. stopping briefly at each corner. 
Table 5.1: Root mean square error for the test trajectories and estimation methods.

\begin{tabular}{c|c|c|c} 
Trajectory & CF & EKF & FK \\
\hline ISO & $0.95 \mathrm{~mm}$ & $0.94 \mathrm{~mm}$ & $1.44 \mathrm{~mm}$ \\
Cube & $0.81 \mathrm{~mm}$ & $0.93 \mathrm{~mm}$ & $0.79 \mathrm{~mm}$
\end{tabular}

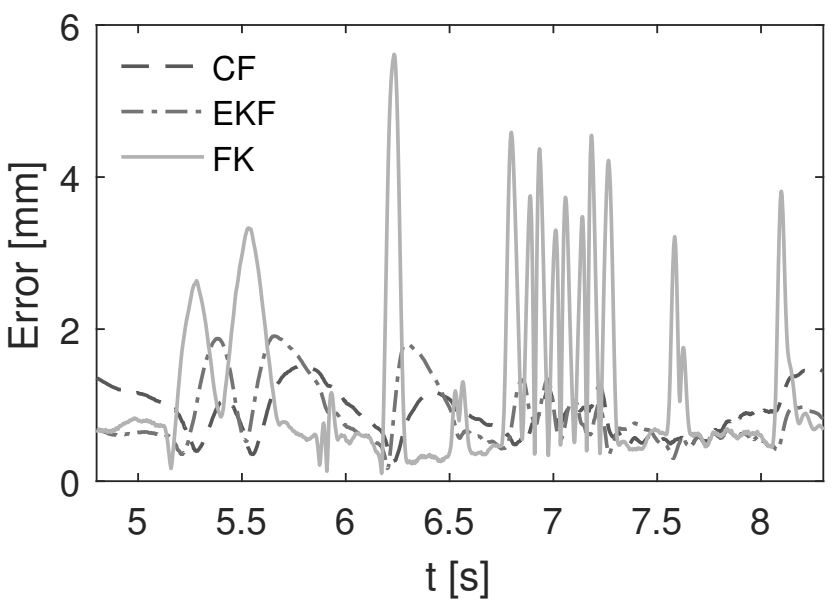

Figure 5.5: Estimation error during a part of the ISO-trajectory. The two wide peaks after $5 \mathrm{~s}$ corresponds to the circle and the rapid succession of peaks corresponds to the series of $90^{\circ}$ turns.

The second traced the edges of a cube with edge length of $30 \mathrm{~mm}$, shown in Figure 5.4, where the target speed was $80 \mathrm{~mm} / \mathrm{s}$ with brief stops in the corners.

In both trajectories the tool orientation was kept constant, as the laser tracking system requires line of sight and cannot measure orientation.

\subsection{Experimental results}

The performance of the estimation methods for the two test trajectories is summarized in Table 5.1, were it can be seen that the CF and the EKF provide similar results in terms of root mean square error, with the CF performing slightly better on the slower of the two trajectories.

The estimates are visualized in Figure 5.2 and Figure 5.4. A close-up of some interesting features from the ISO trajectory is shown in Figure 5.3.

Characteristic parts of the time-error plots for the two trajectories are shown in Figure 5.5 and Figure 5.6 respectively. 


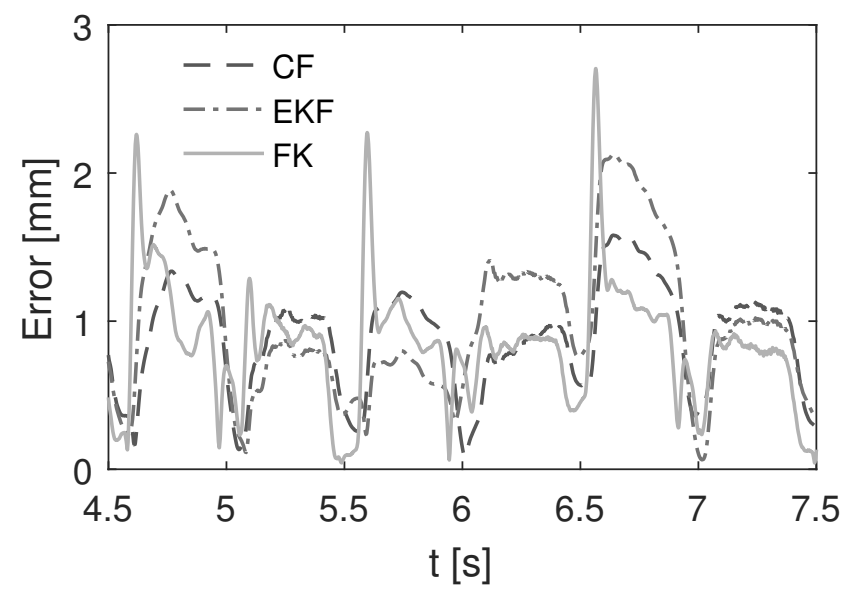

Figure 5.6: Estimation error during part of the cube trajectory, clearly showing the corners as the regions of a low error due to lower speed followed by a larger error due to change in direction.

\subsection{Discussion}

The difference in performance for the forward kinematics estimate, as shown in Table 5.1, between the two trajectories is notable. This is probably due to lower accelerations in the cube trajectory, as the forward kinematics does not capture the dynamic properties excited by acceleration, such as flexibilities in links and gearboxes. This can be supported by comparing the time-error plots and the trajectories, where a clear connection is seen between acceleration and forward kinematics error. E.g. the circle and the rapid succession of $90^{\circ}$-turns in the ISO trajectory can clearly be seen as error peaks for the forward kinematics in Figure 5.5.

The CF and the EKF estimates, using measurements of the acceleration, are not sensitive in the same way. It is, however, clear that several sections can be found where the forward kinematics outperforms both filtering approaches, also in the ISO trajectory, showing how IMU measurement noise is limiting the accuracy of the position estimates.

That the two filtering approaches perform similarly is not surprising given the large ratio between process and measurement noise that the EKF tuning resulted in, as such a ratio is essentially another way of stating the assumptions behind complementary filtering.

In retrospect it is also not surprising that these trajectories, containing parts with quite different characteristics, are not well described by a constant-acceleration model with stationary Gaussian process noise.

As the tool orientation could not be measured, it was kept constant during both trajectories, and nothing conclusive can be said regarding the accuracy of the 
orientation estimates. However, since the orientation estimate is crucial for converting acceleration measurements from the sensor coordinate frame to the world coordinate frame, poor orientation estimates would most likely have had a large negative impact on the position estimates.

\subsection{Conclusions}

By combining the motor angular measurements of an industrial robot with measurements from an IMU mounted on the robot tool, better estimates of the tool position can be achieved compared to using only direct kinematics from motor angle measurements. The forward kinematics estimates deteriorate in parts of the trajectory containing large accelerations, and this is where the filtering approaches offer an improvement.

Between the complementary filter and the extended Kalman filter there was no major difference in performance, the CF performed slightly better during the slower trajectory. This, as well as the large process noise obtained when tuning the EKF, suggests that the statistical properties of the tracked signal are not well described by the chosen motion models and the Gaussian distributions available in the Kalman filtering framework.

When this is the case, using a CF might yield results comparable those of an EKF, while having both a less complex tuning process and lower computational complexity. 


\section{6}

\section{Case study: Feedback linearization compared to LQ-control}

This chapter is a slightly revised version of the paper by Hedberg et al. [2018], which was presented at the 12th IFAC Symposium on Robot Control. It compares two control schemes which are based on two different types of linearization, and introduces a method for doing so in a fair way.

\subsection{Introduction}

A common scheme for control of industrial manipulators is model-based feedforward combined with decentralized PID control of the motor angles. This scheme is relatively simple compared to the many well-known methods for centralized control design offered in literature, but so far the potential benefits of using more complex controllers has not been convincing enough for widespread industrial adoption.

\subsubsection{Motivation}

Motor angles are often the only measurements available for feedback in industrial robots, mainly due to the cost of adding other types of sensors. This has limited the attractiveness of methods that require additional measurements like arm angles or torque.

However, advances in technology and a constant demand for increased performance makes the use of additional sensors an increasingly viable option. As full-state feedback becomes more interesting for industrial applications so does the question of which full-state feedback design method is best suited for use in practice. To answer this question analysis, simulations and real-world experiments are needed. 
The importance of feed-forward to achieve good tracking in robotic manipulators is well established. Therefore, disturbance rejection performance becomes the most important property when comparing different feedback controllers.

When comparing control methods, it is important that the comparison reflects the properties of the respective methods and not the way they were tuned. To that purpose, we introduces a way of choosing an LQ controller for a feedback linearized system such that the LQ loss function is equivalent, at a chosen operating point, to that of an LQ controller designed for a Jacobian linearization in the same operating point.

\subsubsection{Related work}

An overview of control methods for flexible joint robots, including feedback linearization, is provided in the survey paper by Spong [1990]. In a more recent survey by Ozgoli and Taghirad [2006], the authors comment that although there has been many theoretical results published since Spong [1990], adoption of these methods in practice is still scarce.

The benchmark model used in this chapter is introduced in Moberg et al. [2008]. Another benchmark problem for control of flexible joint manipulators is introduced in Hoffmann et al. [2013], together with a controller derived using a linear parameter-varying (LPV) framework. The dissertation by Saupe [2013] is an excellent investigation of LPV control applied to industrial manipulators.

In a recent work Kim and Croft [2017] introduce a control method based on singular perturbation theory and compare it to feedback linearization in experiments on an industrial manipulator. The authors also make the observation that experimental results on feedback linearization are rarely reported in literature.

Brogliato et al. [1995] compares several non-linear control schemes for flexible joint manipulators, but do not include feedback linearization, and analyses them in a common theoretical framework to show structural similarities.

\subsubsection{Contributions}

Feedback linearization for flexible joint robots has been studied extensively from a theoretical point of view in the past, but there are to the authors' best knowledge not many studies comparing it to other control methods in an industrially relevant control problem. This chapter

- introduces a method of designing LQ controllers for Feedback linearization and Jacobian linearization to obtain similar performance in an operating point, and

- compares the two controller types using an industrially relevant benchmark model. 


\subsubsection{Outline}

The benchmark model used for evaluation is introduced in Section 6.2. The control design methods are described in Section 6.3 and Section 6.4 introduces the evaluation methodology. Thereafter the results are presented in Section 6.5 and discussed in Section 6.6.

\subsection{Simulation model}

The simulation model used in this chapter was first introduced in a benchmark problem by Moberg et al. [2008], and has been experimentally verified to well represent the challenge of controlling a typical industrial robotic manipulator. In the benchmark problem, the task is to design a controller with robust performance using only motor angle feedback. Here, we instead study controllers with full state feedback, which are expected to outperform methods that only use actuator positions.

The benchmark model is a two-link flexible joint manipulator, illustrated in Figure 6.1 , and described by the equations

$$
\begin{aligned}
M_{1}\left(q_{a}\right) \ddot{q}_{a}+C\left(q_{a}, \dot{q}_{a}\right)+G\left(q_{a}\right)+D \dot{q}+\tau\left(q_{a}, q_{m}\right) & =0 \\
M_{2} \ddot{q}_{m}-N^{-1} \tau\left(q_{a}, q_{m}\right)+f_{\text {fric }}\left(\dot{q}_{m}\right) & =u
\end{aligned}
$$

where $q$ denotes angle, $a$ arm, and $m$ motor. The model features several realistic properties such as

- non-linear motor friction $f_{\text {fric }}$,

- non-linear spring stiffness $\tau$,

- time delays,

- quantization, and

- measurement noise.

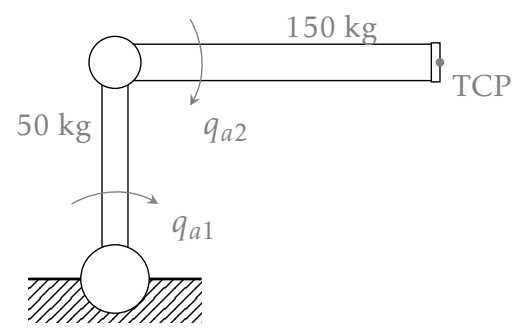

Figure 6.1: Illustration of the robot model at arm configuration $q_{a 1}, q_{a 2}=0$, referred to as operating point 10 in the experimental evaluation. The tool center point (TCP) and link weights are also given. 
The control input $u$ represents motor torques, and is limited by $\left|u_{1}\right| \leq 35 \mathrm{Nm}$, $\left|u_{2}\right| \leq 20 \mathrm{Nm}$. The disturbances acting on the system are summarized in Table 6.1 .

Table 6.1: Disturbances

\begin{tabular}{ll}
\hline Type & Description \\
\hline Motor torques & Frequency sweep from 15 to $0 \mathrm{~Hz}, 1 \mathrm{Nm}$. \\
TCP force & $0.5 \mathrm{~s}$ pulse of $500 \mathrm{~N}$, varying direction. \\
\hline
\end{tabular}

In order to study full-state feedback the model introduced by Moberg et al. [2008] has been extended with measurements of motor velocity as well as arm-side angle and velocity.

\subsection{Control Design}

\subsubsection{Model for control design}

While it is possible to do Feedback linearization for more complex flexible-joint models, see De Luca [1988], we use a simplified model where linear damping and the motor friction are neglected. This gives the model equations

$$
\begin{aligned}
\ddot{q}_{a} & =-M_{1}^{-1}\left(q_{a}\right)\left(C\left(q_{a}, \dot{q}_{a}\right)+G\left(q_{a}\right)+\tau\left(q_{a}, q_{m}\right)\right) \\
\ddot{q}_{m} & =M_{2}^{-1}\left(N^{-1} \tau\left(q_{a}, q_{m}\right)+u\right) .
\end{aligned}
$$

The above simplifications are reasonable as the neglected terms contribute with damping, and the approximation will thus be conservative in the sense that the real system is better damped, see Khalil [2002]. Introduce the notation

$$
P: \dot{x}=f(x)+B u, \quad x=\left[\begin{array}{llll}
q_{a}^{T} & \dot{q}_{a}^{T} & q_{m}^{T} & \dot{q}_{m}^{T}
\end{array}\right]^{T}
$$

for the plant used for control design and its states.

Assumption 6.1. Furthermore, assume that $f(x)$ includes gravity compensation such that all stationary joint configurations $q_{a}$ are equilibrium points for the system $P$.

\subsubsection{LQ Control}

Linear Quadratic (LQ) control is a well-known method for control of linear systems, and is briefly introduced below. See e.g. Franklin et al. [2002] for further details. Given a controllable linear system and a quadratic loss function

$$
V(x, u)=\left[\begin{array}{l}
x \\
u
\end{array}\right]^{T}\left[\begin{array}{cc}
Q & N / 2 \\
N / 2 & R
\end{array}\right]\left[\begin{array}{l}
x \\
u
\end{array}\right]
$$


a state feedback $u=-L x$ can always be found such that

$$
\int_{0}^{\infty} V(x, u) d t
$$

is minimized, given that the penalty matrices $Q, R$, and $N$ are chosen to ensure that the loss function (6.3) is positive semi-definite. The cross term matrix $N$ is seldom used in practice, as it is often hard to interpret intuitively.

\subsubsection{Jacobian Linearization}

In order to apply LQ design to the non-linear plant $P$, it is approximated by a linear plant $P_{\delta}$. The approximation will be good in some neighbourhood of the equilibrium point $x_{0}$, and the linear system can be written

$$
P_{\delta}: \dot{x}=f_{x}^{\prime}\left(x_{0}\right) x_{\delta}+B u, \quad x_{\delta}=x-x_{0}
$$

where $f_{x}^{\prime}\left(x_{0}\right)$ is the Jacobian matrix of $f$ evaluated at $x_{0}$. This is one of the most fundamental methods of designing controllers for non-linear plants.

Now, introduce the following loss function

$$
V_{\delta}=x_{\delta}^{T} Q x_{\delta}+u^{T} R u
$$

which will be used in designing an LQ controller

$$
K_{\delta}: u=-L_{\delta} x_{\delta}
$$

for the plant model $P_{\delta}$.

Assumption 6.2. Restrict the choice of $Q$ to only penalize the states $q_{a}^{T}$ and $\dot{q}_{a}^{T}$. This simplifies derivations and is reasonable from an application viewpoint, as end-effector movement depends directly on the arm angles.

\subsubsection{Feedback Linearization}

Some classes of non-linear systems can be turned into a linear system, without approximation, by a non-linear state transform and feedback. This is called Feedback linearization or sometimes Exact linearization (e.g. Kreutz [1989]) and can be found in standard textbooks on non-linear control such as Khalil [2002]. Even when it is not possible to render a system exactly linear, feedback may still render it linear enough that linear control methods can be applied successfully.

For example, terms that have a damping effect, like friction, can often be left out of the linearizing feedback with good results, as described by e.g. Slotine and Li [1991].

The state transform for feedback linearization of $P$ and the resulting states can be written

$$
y=T(x), \quad y=\left[\begin{array}{llll}
q_{a}^{T} & \dot{q}_{a}^{T} & \ddot{q}_{a}^{T} & q_{a}^{(3) T}
\end{array}\right]^{T}
$$


and can be derived by calculating the higher derivatives of $q_{a}$, preferably with a computer algebra system. The non-linear feedback

$$
u=\alpha(x)+\beta(x) v
$$

where $v$ is an artificial input, can then be derived by solving $q_{a}^{(4)}(x, u)=v$ for $u$. Using this feedback gives the closed loop

$$
P_{F}: \dot{x}=f(x)+B(\alpha(x)+\beta(x) v),
$$

which is a linear system in the $y$-coordinates

$$
P_{F}: \dot{y}=A_{F} y+B_{F} v
$$

Note that while $P_{\delta}$ is a linear approximation of $P$, the feedback linearized system $P_{F}$ is a linear plant with different dynamics. Also note that the arm angles and arm angular velocity states $q_{a}, \dot{q}_{a}$ are unchanged under the transformation,

$$
x=\left[\begin{array}{llll}
q_{a}^{T} & \dot{q}_{a}^{T} & * & *
\end{array}\right]^{T} \Longrightarrow T(x)=\left[\begin{array}{llll}
q_{a}^{T} & \dot{q}_{a}^{T} & * & *
\end{array}\right]^{T} .
$$

The linear plant (6.11) can now be controlled using any control method for linear systems.

However, due to the non-linear transformation, penalizing the magnitude of the artificial control inputs $v$ does not translate directly to a penalty on the real control signals $u$. This is one of the challenges of using feedback linearization in practice.

\subsubsection{Equivalent LQ Design}

Let the deviation of $y$ from a desired equilibrium point $y_{0}=T\left(x_{0}\right)$ be denoted $y_{\delta}$, and introduce the LQ controller

$$
K_{F}: v=-L_{F} y_{\delta}
$$

for use with the linearizing feedback (6.9). Use the feedback linearization variables $y_{\delta}$ and $v$ to write the corresponding loss function as

$$
V_{F}\left(y_{\delta}, v\right)=y_{\delta}^{T} Q_{F} y_{\delta}+v^{T} R_{F} v+y_{\delta}^{T} N_{F} v \text {. }
$$

Our interest now lies in choosing $V_{F}$ so that the closed loop with $K_{F}$ has equivalent performance to that of $K_{\delta}$ at the operating point $x_{0}$. To that end, consider that the loss function $V_{\delta}$, given in (6.6), dictates the behaviour of the closed loop under $K_{\delta}$.

Proposition 6.3. The quadratic function $V_{F}$ can be chosen to equal the second order Taylor expansion of $V_{\delta}$ at $x_{0}$.

Proof: Consider the Taylor expansion of the feedback law (6.9) at the equilibrium 
point $x_{0}$ given by

$$
u=\alpha(x)+\beta(x) v=\alpha_{x}^{\prime}\left(x_{0}\right) x_{\delta}+\beta\left(x_{0}\right) v+\mathcal{O}\left(\left\|x_{\delta}\right\|^{2}\right)
$$

where the $\mathcal{O}$ term uses the fact that

$$
v=-L_{F} y_{\delta}=-L_{F} T_{x}^{\prime}\left(x_{0}\right) x_{\delta}+\mathcal{O}\left(\left\|x_{\delta}\right\|^{2}\right) \text {. }
$$

Substitute (6.15) into $V_{\delta}$ given by (6.6) to obtain

$$
V_{\delta}=x_{\delta}^{T}(Q+\tilde{Q}) x_{\delta}+v^{T} \tilde{R} v+x_{\delta}^{T} \tilde{N} v+\mathcal{O}\left(\left\|x_{\delta}\right\|^{3}\right),
$$

where

$$
\begin{aligned}
\tilde{Q} & =\alpha_{x}^{\prime}\left(x_{0}\right)^{T} R \alpha_{x}^{\prime}\left(x_{0}\right), \\
\tilde{R} & =\beta\left(x_{0}\right)^{T} R \beta\left(x_{0}\right), \\
\tilde{N} & =2 \alpha_{x}^{\prime}\left(x_{0}\right)^{T} R \beta\left(x_{0}\right) .
\end{aligned}
$$

From (6.8) and Assumption 6.2 in Section 6.3.3 that $Q$ only penalizes the states $q_{a}^{T}$ and $\dot{q}_{a}^{T}$ we have

$$
x_{\delta}^{T} Q x_{\delta}=y_{\delta}^{T} Q y_{\delta} .
$$

For notational brevity introduce

$$
H=\left(T_{x}^{\prime}\left(x_{0}\right)\right)^{-1} \Longrightarrow x_{\delta}=H y_{\delta}+\mathcal{O}\left(\left\|x_{\delta}\right\|^{2}\right)
$$

and substitute both (6.19) and (6.20) into (6.17) to obtain

$$
\begin{aligned}
V_{\delta}= & y_{\delta}^{T}\left(Q+H^{T} \tilde{Q} H\right) y_{\delta}+v^{T} \tilde{R} v+y_{\delta}^{T} H^{T} \tilde{N} v \\
& +\mathcal{O}\left(\left\|x_{\delta}\right\|^{3}\right) .
\end{aligned}
$$

Now, by comparing (6.21) with (6.14) it is clear that by using the weight matrices

$$
Q_{F}=Q+H^{T} \tilde{Q} H, \quad R_{F}=\tilde{R}, \quad N_{F}=H^{T} \tilde{N},
$$

the loss function $V_{F}$ becomes a second order approximation of $V_{\delta}$ around the equilibrium point $x_{0}$.

Using the above choice of weight matrices, the controller $K_{F}$ can be expected to result in the same behaviour as $K_{\delta}$ for small deviations from $x_{0}$, since the behaviour of an LQ controller is entirely dictated by the choice of loss function $V$, and the difference between the loss functions $V_{F}$ and $V_{\delta}$ vanishes at $x_{0}$.

This approximative equivalence is of course only valid around the chosen equilibrium point, and deriving analytically what it means in terms of real world performance is difficult. Therefore we propose a simulated experimental evaluation.

\subsubsection{Simplification of Equivalent LQ Design}

One benefit of the method proposed in Section 6.3.5 is that it penalizes $v$ in such a way as to avoid large control inputs $u$, thus solving one of the challenges of 
using feedback linearization. This benefit can also be obtained with the simpler choice

$$
Q_{F}=Q, \quad R_{F}=\tilde{R}, \quad N_{F}=0,
$$

which disregards the contribution of $\alpha(x)$ to $u$ in (6.9), and thus simplifies calculations considerably.

In practice, the choice (6.23) was found to improve results compared to the choice (6.22), especially for large disturbances, and is therefore used in the following experimental evaluation.

\subsection{Experimental evaluation}

\subsubsection{Implementation and simulation}

The non-linear transform $T(x)$ and related expressions $\alpha(x), \beta(x)$ and $H\left(x_{0}\right)$ were derived from the state equations (6.1) using computer algebra software. This process also generated functions for use in the simulation. The Jacobian matrices used in $P_{\delta}$ were obtained in the same manner.

Moberg et al. [2008] provides the benchmark models as a robot model written in $\mathrm{C}$ and a control system designed in Simulink. This was extended with additional measurements and measurement noise.

\subsubsection{Controller tuning}

The weight matrices $Q$ and $R$ defining the loss function were chosen to be diagonal, and suitable values were found through an extensive grid search over weight matrices at different working points and noise levels.

Based on the grid search, two tunings, referred to as the high gain tuning (HG) and the low gain tuning (LG), were selected for further analysis.

\subsubsection{Operating points}

The system was evaluated at 18 different operating points, corresponding to the arm angles

$$
q_{a}^{n}=\left[\begin{array}{c}
0 \\
-100^{\circ}+n \cdot 10^{\circ}
\end{array}\right], n=1 \ldots 18 .
$$

Note that in the coordinate system of the benchmark model, $q_{a 1}=0$ signifies the first link is standing upright, and $q_{a 2}=0$ that the second link is rotated $90^{\circ}$ with respect to the first link, as illustrated in Figure (6.1).

\subsubsection{Performance measure}

The benchmark problem introduced in Moberg et al. [2008] includes a performance measure chosen to describe desirable performance for an industrial ma- 


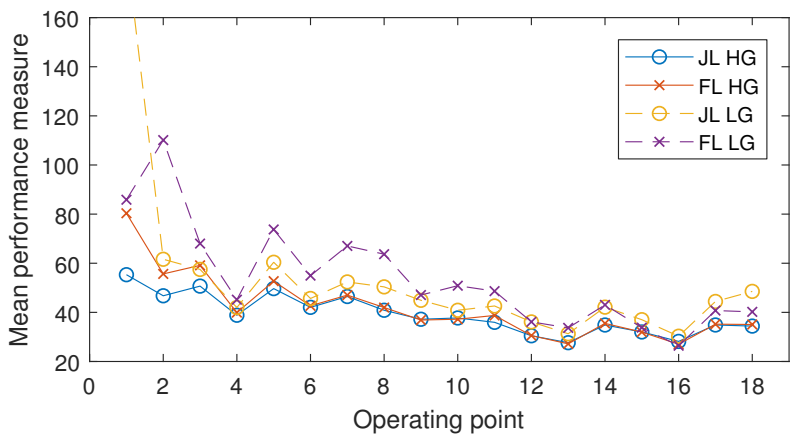

Figure 6.2: The performance measure obtained in different working points. Lower is better. Especially for the high gain tuning, the two controller schemes have similar performance.

nipulator. The measure is a weighted sum of the maximum value of

- motor torque due to measurement noise,

- overall motor torque,

- tool position error due to external force disturbance,

- settling time after force disturbance, and

- tool position error due to motor disturbance.

All features are normalized with respect to a target performance. The tool position errors and the settling time together make up $90 \%$ of the performance measure.

\subsection{Results}

The performance measure obtained from simulations in the different working points is illustrated in Figure 6.2.

Representative examples of system responses to motor disturbances and external force disturbances are shown in Figure 6.3 and 6.4 respectively.

\subsection{Discussion}

\subsubsection{Similarity}

Tuning LQ controllers for Feedback linearization and Jacobian linearization respectively, the proposed method seems to give similar behaviour. Both in the sense that the two controller types achieve similar performance measures, as shown in Figure 6.2. But also in the sense that the system response to disturbances is strikingly similar in many cases, as illustrated by Figures 6.3 and 6.4. 

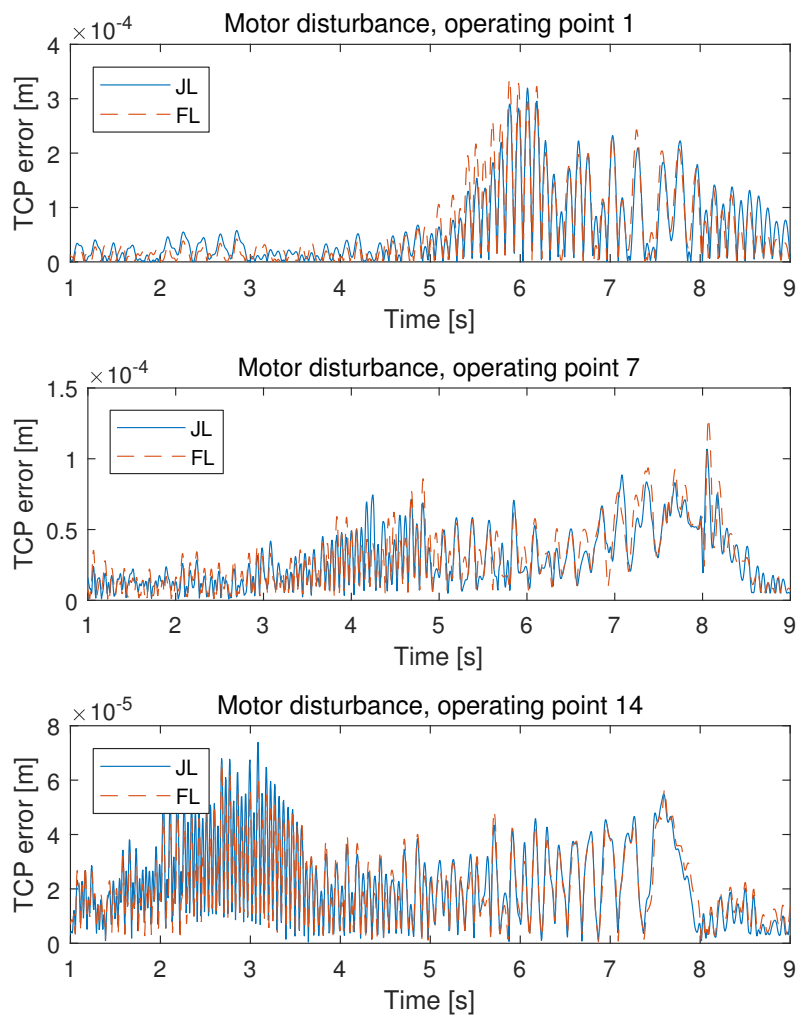

Figure 6.3: Tool centre point (TCP) position error due to motor torque chirp disturbance, using the high-gain configuration of the controllers. The two controller schemes show clear similarities. 

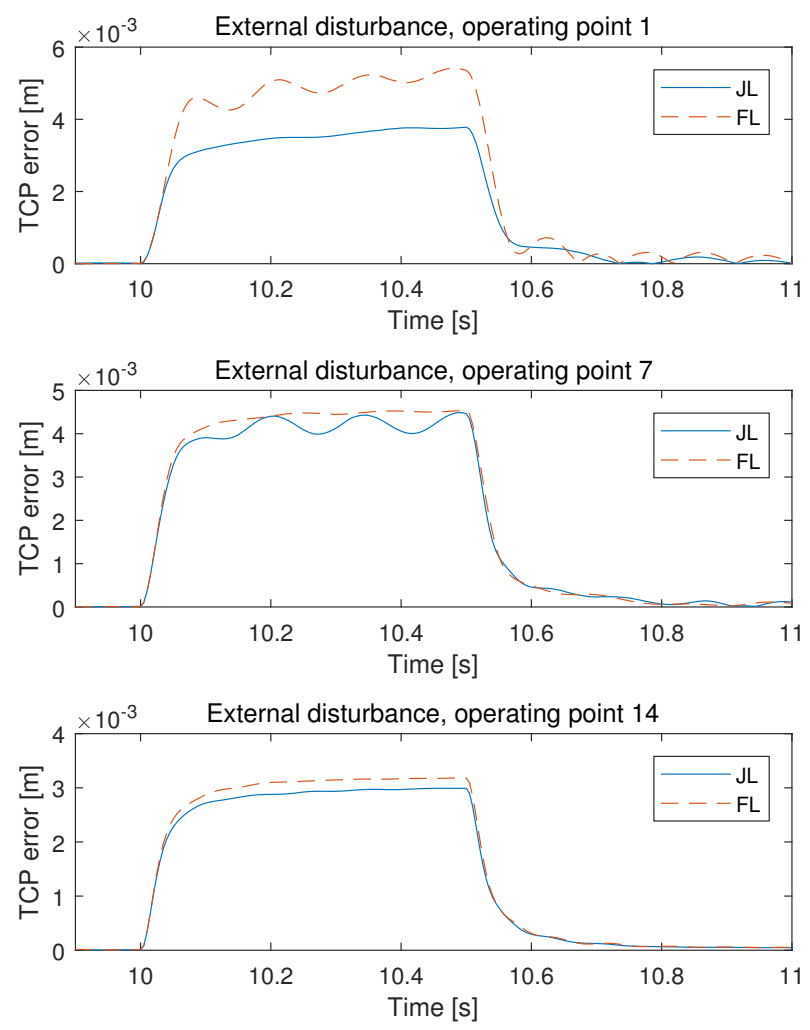

Figure 6.4: Tool centre point (TCP) position error due to external force acting on the end-effector, using the high-gain configuration of the controllers.

From the system responses it is also clear that the two controller schemes result in closed loop systems with similar performance.

The similarity is stronger when using higher gains, which can be expected as a more aggressive controller will keep the system closer to the operating point used for controller design. By the same reasoning, it is not surprising that the similarity is lower in the operating points close to the singular arm configuration $q_{a 1}=0, q_{a 2}=-90$, where disturbance rejection is more challenging.

\subsubsection{Impact of non-linear spring}

Due to the non-linear spring stiffness, a Jacobian linearization around an equilibrium point will in most cases underestimate the spring stiffness and therefore result in a larger feedback gain. This effect can be seen in the end-effector error due to the external force disturbance, where the Jacobian linearization consistently outperforms the Feedback linearization as exemplified in Figure 6.4. The slight overall performance advantage of the Jacobian linearization could also be 
due to this fact.

\subsubsection{Considerations for control designers}

From an engineering point of view, the Jacobian linearization scheme is significantly less demanding, both in implementation and in computational resources. The small differences in performance between the two schemes therefore suggest that for centralized disturbance rejection during trajectory tracking, a gain scheduling scheme based on Jacobian linearization might be a more efficient use of computational resources, which are in general a limiting factor on robot controllers.

The experimental evaluation also showed that quantization of arm-side measurements is a concern at the level of accuracy required in industrial manipulators. A 16-bit quantization of arm angles can give TCP errors on the order of $0.1 \mathrm{~mm}$ in the present model. This problem is less pronounced for motor side measurements, since the gear ratio of $n=200$ means motor measurements translated to arm side gain $\log _{2}(n) \approx 7.6$ bits of resolution.

\subsection{Conclusions}

Feedback linearization was compared to Jacobian linearization for disturbance rejection using an LQ controller. To that end, a method for equivalent controller tuning was introduced. Experiments on an industrially relevant benchmark model did not show any clear benefits of using Feedback linearization over Jacobian linearization.

Further studies are needed to compare the robustness properties of the two control schemes. Robustness to model errors is important, but to use full state feedback in practice, robustness to imperfect state estimates is crucial. 


\section{Bibliography}

Robotics ABB. Datasheet IRB4600 Industrial Robot, 2016.

W. G. Anderson and E. H. Fritze. Instrument approach system steering computer. Proceedings of the IRE, 41(2):219-228, 1953.

Karl J. Åström and Richard M. Murray. Feedback Systems: An Introduction for Scientists and Engineers. Princeton University Press, Princeton, 2008. ISBN 978-0-691-13576-2.

Karl J. Åström and Björn Wittenmark. Computer Controlled Systems: Theory and Design. Prentice-Hall Information and System Sciences Series. PrenticeHall, Englewood Cliffs, N.J, 1984. ISBN 978-0-13-164319-2.

Johanna Wallén Axehill, Isolde Dressler, Svante Gunnarsson, Anders Robertsson, and Mikael Norrlöf. Estimation-based ILC applied to a parallel kinematic robot. Control Engineering Practice, 33:1-9, December 2014. ISSN 09670661. doi: 10.1016/j.conengprac.2014.08.008.

John Bechhoefer. Feedback for physicists: A tutorial essay on control. Reviews of Modern Physics, 77(3):783, 2005.

B. Wayne Bequette. Process Control: Modeling, Design, and Simulation. PrenticeHall International Series in the Physical and Chemical Engineering Sciences. Prentice Hall PTR, Upper Saddle River, N.J, 2003. ISBN 978-0-13-353640-9.

Bernard Brogliato, Romeo Ortega, and Rogelio Lozano. Global tracking controllers for flexible-joint manipulators: A comparative study. Automatica, 31 (7):941-956, 1995.

Coleman Brosilow and Martin Tong. Inferential control of processes: Part II. The structure and dynamics of inferential control systems. AIChE Journal, 24(3): 492-500, 1978. ISSN 1547-5905. doi: 10.1002/aic.690240314.

R. G. Brown. Integrated Navigation Systems and Kalman Filtering: A Perspective. Navigation, 19(4):355-362, December 1972. ISSN 00281522. doi: 10.1002/j. 2161-4296.1972.tb01706.x. 
Robert Grover Brown and Patrick Y. C. Hwang. Introduction to Random Signals and Applied Kalman Filtering: With MATLAB Exercises and Solutions. Wiley, New York, 3rd ed edition, 1997. ISBN 978-0-471-12839-7.

Mario R. Cesca and Jacinto L. Marchetti. IMC design of cascade control. In Computer Aided Chemical Engineering, volume 20, pages 1243-1248. Elsevier, 2005. ISBN 978-0-444-51987-0. doi: 10.1016/S1570-7946(05)80049-5.

Chi-Tsong Chen. Linear System Theory and Design. The Oxford Series in Electrical and Computer Engineering. Oxford University Press, New York, 3rd ed edition, 1999. ISBN 978-0-19-511777-6 978-0-19-511778-3.

Wenjie Chen and Masayoshi Tomizuka. Direct Joint Space State Estimation in Robots With Multiple Elastic Joints. IEEE/ASME Transactions on Mechatronics, 19(2):697-706, April 2014. ISSN 1083-4435, 1941-014X. doi: 10.1109/ TMECH.2013.2255308.

Roger C. Conant and W. Ross Ashby. Every good regulator of a system must be a model of that system. International Journal of Systems Science, 1(2):89-97, October 1970. ISSN 0020-7721. doi: 10.1080/00207727008920220.

Jean-Pierre Corriou. Process Control: Theory and Applications. Springer, London ; New York, 2004. ISBN 978-1-85233-776-6.

A. De Luca. Dynamic control of robots with joint elasticity. In 1988 IEEE International Conference on Robotics and Automation Proceedings, pages 152-158 vol.1, April 1988. doi: 10.1109/ROBOT.1988.12040.

Richard C. Dorf and Robert H. Bishop. Modern Control Systems. Pearson/Prentice Hall, Upper Saddle River, NJ, 11th ed edition, 2008. ISBN 978-013-227028-1.

Michel Fliess and Cédric Join. Model-free control. International Journal of Control, 86(12):2228-2252, December 2013. ISSN 0020-7179. doi: 10.1080/ 00207179.2013 .810345 .

B. A. Francis and W. M. Wonham. The internal model principle for linear multivariable regulators. Applied Mathematics and Optimization, 2(2):170-194, June 1975. ISSN 1432-0606. doi: 10.1007/BF01447855.

Paul M. Frank. Entwurf von Regelkreisen Mit Vorgeschriebenem Verhalten. Wissenschaft Und Technik : Taschenausgaben. Braun, Karlsruhe, 1974. ISBN 9783-7650-2016-2.

Gene F. Franklin, J. David Powell, and Abbas Emami-Naeini. Feedback Control of Dynamic Systems. Prentice Hall, Upper Saddle River, NJ, 4th ed edition, 2002. ISBN 978-0-13-032393-4.

Carlos E. Garcia and Manfred Morari. Internal model control. A unifying review and some new results. Industrial \& Engineering Chemistry Process Design and Development, 21(2):308-323, April 1982. ISSN 0196-4305. doi: 10.1021/ i200017a016. 
Carlos E. García, David M. Prett, and Manfred Morari. Model predictive control: Theory and practice-A survey. Automatica, 25(3):335-348, May 1989. ISSN 0005-1098. doi: 10.1016/0005-1098(89)90002-2.

Paul Gewirtz. On "I Know It When I See It". The Yale Law Journal, 105(4):1023, January 1996. ISSN 00440094. doi: 10.2307/797245.

Torkel Glad and Lennart Ljung. Control Theory. CRC Press, 2000. ISBN 978-14822-6816-4. doi: 10.1201/9781315274737.

Graham Clifford Goodwin, Stefan F. Graebe, and Mario E. Salgado. Control System Design. Prentice Hall, Upper Saddle River, NJ, 2001. ISBN 978-0-13958653-8.

Fredrik Gustafsson. Statistical Sensor Fusion. Studentlitteratur, Lund, 2012. ISBN 978-91-44-07732-1.

Erik Hedberg, Johan Norén, Mikael Norrlöf, and Svante Gunnarsson. Industrial Robot Tool Position Estimation using Inertial Measurements in a Complementary Filter and an EKF. 2017.

Erik Hedberg, Mikael Norrlöf, Stig Moberg, and Svante Gunnarsson. Comparing feedback linearization and jacobian linearization for LQ control of an industrial manipulator. In Proccedings of the 12TH IFAC SYMPOSIUM ON ROBOT CONTROL, 2018.

Erik Hedberg, Johan Löfberg, and Anders Helmersson. A pedagogical path from the internal model principle to Youla-Kucera parametrization. In 21st IFAC World Congress 2020, 2020.

Leah Henderson. The problem of induction. In Edward N. Zalta, editor, The Stanford Encyclopedia of Philosophy. Metaphysics Research Lab, Stanford University, spring 2020 edition, 2020.

C. Hoffmann, S. M. Hashemi, H. S. Abbas, and H. Werner. Benchmark problem Nonlinear control of a 3-DOF robotic manipulator. In 52nd IEEE Conference on Decision and Control, pages 5534-5539, December 2013. doi: 10.1109/ CDC.2013.6760761.

Jeroen Hol. Sensor fusion and calibration of inertial sensors, vision, ultrawideband and GPS. PhD thesis, Department of Electrical Engineering, Linköping University, Linköping, 2011.

Isaac M Horowitz. Synthesis of Feedback Systems. Academic Press, New York, 1963.

IFR. World Robotics 2019 - Industrial Robots. Technical report, International Federation of Robotics, Frankfurt am Main, Germany, 2019.

ISO 8373. Robots and robotic devices - Vocabulary. Standard ISO 8373:2012, International Organization for Standardization, Geneva, Switzerland, 2012. 
ISO 9283. Manipulating industrial robots - Performance criteria and related test methods. Standard ISO 9283:1998, International Organization for Standardization, Geneva, Switzerland, 1998.

Austin Jensen, Cal Coopmans, and YangQuan Chen. Basics and guidelines of complementary filters for small UAS navigation. In Unmanned Aircraft Systems (ICUAS), 2013 International Conference On, pages 500-507. IEEE, 2013.

Hassan Khalil. Nonlinear Systems. Prentice Hall, January 2002. ISBN 978-0-13122740-8.

J. Kim and E. A. Croft. Full-State Tracking Control for Flexible Joint Robots With Singular Perturbation Techniques. IEEE Transactions on Control Systems Technology, PP(99):1-11, 2017. ISSN 1063-6536. doi: 10.1109/TCST.2017. 2756962.

Kenneth Kreutz. On manipulator control by exact linearization. IEEE Transactions on Automatic Control, 34(7):763-767, July 1989. ISSN 0018-9286. doi: 10.1109/9.29408.

Vladimír Kučera. Stability of Discrete Linear Feedback Systems. IFAC Proceedings Volumes, 8(1, Part 1):573-578, August 1975. ISSN 1474-6670. doi: 10.1016/S1474-6670(17)67787-5.

Leica Geosystems. 2016.

Lennart Ljung. System Identification: Theory for the User. Prentice Hall Information and System Sciences Series. Prentice Hall PTR, Upper Saddle River, NJ, 2nd ed edition, 1999. ISBN 978-0-13-656695-3.

Jan Marian Maciejowski. Multivariable Feedback Design. Electronic Systems Engineering Series. Addison-Wesley, Wokingham, England ; Reading, Mass, 1989. ISBN 978-0-201-18243-9.

Thomas E. Marlin. Process Control: Designing Processes and Control Systems for Dynamic Perfomance. McGraw-Hill, Boston, 2nd ed edition, 2000. ISBN 978-0-07-039362-2.

Peter S. Maybeck. Stochastic Models, Estimation and Control. Number v. 141 in Mathematics in Science and Engineering. Academic Press, New York, 1979. ISBN 978-0-12-480701-3.

McKinsey \& Company. Industrial robotics: Insights into the sector's future growth dynamics. Technical report, 2019.

Stig Moberg. Modeling and Control of Flexible Manipulators. PhD Thesis, Linköping University, 2010.

Stig Moberg, Jonas Öhr, and Svante Gunnarsson. A Benchmark Problem for Robust Control of a Multivariable Nonlinear Flexible Manipulator. IFAC Proceedings Volumes, 41:1206-1211, 2008. ISSN 1474-6670. doi: 10.3182/ 20080706-5-KR-1001.00208. 
Manfred Morari and Evanghelos Zafiriou. Robust Process Control. Prentice Hall, Englewood Cliffs, N.J, 1989. ISBN 978-0-13-782153-2.

M. Nakao, K. Ohnishi, and K. Miyachi. A Robust decentralized joint control based on interference estimation. In 1987 IEEE International Conference on Robotics and Automation Proceedings, volume 4, pages 326-331, March 1987. doi: 10.1109/ROBOT.1987.1087996.

Johan Norén. IMU-baserad skattning av verktygets position och orientering hos industrirobot. 2014.

Roberto Oboe. How disturbance observer changed my life. In 2018 IEEE 15th International Workshop on Advanced Motion Control (AMC), pages 13-20, March 2018. doi: 10.1109/AMC.2019.8371055.

Björn Olofsson, Jacob Antonsson, Henk G. Kortier, Bo Bernhardsson, Anders Robertsson, and Rolf Johansson. Sensor Fusion for Robotic Workspace State Estimation. IEEE/ASME Transactions on Mechatronics, 21(5):2236-2248, October 2016. ISSN 1083-4435, 1941-014X. doi: 10.1109/TMECH.2015.2506041.

Winfried Oppelt. Kleines Handbuch technischer Regelvorgänge. Verlag Chemie, Berlin, 4., neubearb. u. erw. aufl edition, 1964.

S. Ozgoli and H. D. Taghirad. A Survey on the Control of Flexible Joint Robots. Asian Journal of Control, 8(4):1-15, December 2006.

Philip Roan, Nikhil Deshpande, Yizhou Wang, and Benjamin Pitzer. Manipulator state estimation with low cost accelerometers and gyroscopes. In 2012 IEEE/RSJ International Conference on Intelligent Robots and Systems, pages 4822-4827. IEEE, 2012.

Florian Saupe. Linear Parameter Varying Control Design for Industrial Manipulators. Ph.D. Thesis, Universität Hamburg-Harburg, 2013.

Daniele Semino and Alessandro Brambilla. An Efficient Structure for Parallel Cascade Control. Industrial \& Engineering Chemistry Research, 35(6):18451852, January 1996. ISSN 0888-5885, 1520-5045. doi: 10.1021/ie950710r.

Sensonor AS. Datasheet STIM300. 2016.

Jean-Jacques E. Slotine and Weiping Li. Applied nonlinear control. Prentice Hall, 1991.

Michael Smith. The Humean Theory of Motivation. Mind, 96(381):36-61, 1987. ISSN 0026-4423.

Mark W. Spong. Control of flexible joint robots: A survey. Coordinated Science Laboratory Report no. UILU-ENG 90-2203, 1990.

Jan C. Willems. The Behavioral Approach to Open and Interconnected Systems. IEEE Control Systems, 27(6):46-99, December 2007. ISSN 1066-033X, 1941000X. doi: 10.1109/MCS.2007.906923. 
Walter H. Wirkler. Aircraft course stabilizing means, April 1951.

William A Wolovich. Linear Multivariable Systems. Springer Verlag, 1974. ISBN 9781461263920.

W. M. Wonham. Towards an Abstract Internal Model Principle. IEEE Transactions on Systems, Man, and Cybernetics, SMC-6(11):735-740, November 1976. ISSN 0018-9472. doi: 10.1109/TSMC.1976.4309444.

D. Youla, H. Jabr, and J. Bongiorno. Modern Wiener-Hopf design of optimal controllers-Part II: The multivariable case. IEEE Transactions on Automatic Control, 21(3):319-338, June 1976. ISSN 0018-9286, 1558-2523, 2334-3303. doi: 10.1109/TAC.1976.1101223. 


\section{Licentiate Theses \\ Division of Automatic Control \\ Linköping University}

P. Andersson: Adaptive Forgetting through Multiple Models and Adaptive Control of Car Dynamics. Thesis No. 15, 1983.

B. Wahlberg: On Model Simplification in System Identification. Thesis No. 47, 1985.

A. Isaksson: Identification of Time Varying Systems and Applications of System Identification to Signal Processing. Thesis No. 75, 1986.

G. Malmberg: A Study of Adaptive Control Missiles. Thesis No. 76, 1986.

S. Gunnarsson: On the Mean Square Error of Transfer Function Estimates with Applications to Control. Thesis No. 90, 1986.

M. Viberg: On the Adaptive Array Problem. Thesis No. 117, 1987.

K. Ståhl: On the Frequency Domain Analysis of Nonlinear Systems. Thesis No. 137, 1988.

A. Skeppstedt: Construction of Composite Models from Large Data-Sets. Thesis No. 149, 1988.

P. A. J. Nagy: MaMiS: A Programming Environment for Numeric/Symbolic Data Processing. Thesis No. 153, 1988.

K. Forsman: Applications of Constructive Algebra to Control Problems. Thesis No. 231, 1990.

I. Klein: Planning for a Class of Sequential Control Problems. Thesis No. 234, 1990.

F. Gustafsson: Optimal Segmentation of Linear Regression Parameters. Thesis No. 246, 1990.

H. Hjalmarsson: On Estimation of Model Quality in System Identification. Thesis No. 251, 1990.

S. Andersson: Sensor Array Processing; Application to Mobile Communication Systems and Dimension Reduction. Thesis No. 255, 1990.

K. Wang Chen: Observability and Invertibility of Nonlinear Systems: A Differential Algebraic Approach. Thesis No. 282, 1991.

J. Sjöberg: Regularization Issues in Neural Network Models of Dynamical Systems. Thesis No. 366, 1993.

P. Pucar: Segmentation of Laser Range Radar Images Using Hidden Markov Field Models. Thesis No. 403, 1993.

H. Fortell: Volterra and Algebraic Approaches to the Zero Dynamics. Thesis No. 438, 1994.

T. McKelvey: On State-Space Models in System Identification. Thesis No. 447, 1994.

T. Andersson: Concepts and Algorithms for Non-Linear System Identifiability. Thesis No. 448, 1994.

P. Lindskog: Algorithms and Tools for System Identification Using Prior Knowledge. Thesis No. 456, 1994.

J. Plantin: Algebraic Methods for Verification and Control of Discrete Event Dynamic Systems. Thesis No. 501, 1995.

J. Gunnarsson: On Modeling of Discrete Event Dynamic Systems, Using Symbolic Algebraic Methods. Thesis No. 502, 1995.

A. Ericsson: Fast Power Control to Counteract Rayleigh Fading in Cellular Radio Systems. Thesis No. 527, 1995.

M. Jirstrand: Algebraic Methods for Modeling and Design in Control. Thesis No. 540, 1996.

K. Edström: Simulation of Mode Switching Systems Using Switched Bond Graphs. Thesis No. 586, 1996. 
J. Palmqvist: On Integrity Monitoring of Integrated Navigation Systems. Thesis No. 600, 1997.

A. Stenman: Just-in-Time Models with Applications to Dynamical Systems. Thesis No. 601, 1997.

M. Andersson: Experimental Design and Updating of Finite Element Models. Thesis No. 611, 1997.

U. Forssell: Properties and Usage of Closed-Loop Identification Methods. Thesis No. 641, 1997.

M. Larsson: On Modeling and Diagnosis of Discrete Event Dynamic systems. Thesis No. 648, 1997.

N. Bergman: Bayesian Inference in Terrain Navigation. Thesis No. 649, 1997.

V. Einarsson: On Verification of Switched Systems Using Abstractions. Thesis No. 705, 1998.

J. Blom, F. Gunnarsson: Power Control in Cellular Radio Systems. Thesis No. 706, 1998.

P. Spångéus: Hybrid Control using LP and LMI methods - Some Applications. Thesis No. 724, 1998.

M. Norrlöf: On Analysis and Implementation of Iterative Learning Control. Thesis No. 727, 1998.

A. Hagenblad: Aspects of the Identification of Wiener Models. Thesis No. 793, 1999.

F. Tjärnström: Quality Estimation of Approximate Models. Thesis No. 810, 2000.

C. Carlsson: Vehicle Size and Orientation Estimation Using Geometric Fitting. Thesis No. 840, 2000.

J. Löfberg: Linear Model Predictive Control: Stability and Robustness. Thesis No. 866, 2001.

O. Härkegård: Flight Control Design Using Backstepping. Thesis No. 875, 2001.

J. Elbornsson: Equalization of Distortion in A/D Converters. Thesis No. 883, 2001.

J. Roll: Robust Verification and Identification of Piecewise Affine Systems. Thesis No. 899, 2001.

I. Lind: Regressor Selection in System Identification using ANOVA. Thesis No. 921, 2001.

R. Karlsson: Simulation Based Methods for Target Tracking. Thesis No. 930, 2002.

P.-J. Nordlund: Sequential Monte Carlo Filters and Integrated Navigation. Thesis No. 945, 2002.

M. Östring: Identification, Diagnosis, and Control of a Flexible Robot Arm. Thesis No. $948,2002$.

C. Olsson: Active Engine Vibration Isolation using Feedback Control. Thesis No. 968, 2002.

J. Jansson: Tracking and Decision Making for Automotive Collision Avoidance. Thesis No. 965, 2002.

N. Persson: Event Based Sampling with Application to Spectral Estimation. Thesis No. 981, 2002.

D. Lindgren: Subspace Selection Techniques for Classification Problems. Thesis No. 995, 2002.

E. Geijer Lundin: Uplink Load in CDMA Cellular Systems. Thesis No. 1045, 2003.

M. Enqvist: Some Results on Linear Models of Nonlinear Systems. Thesis No. 1046, 2003.

T. Schön: On Computational Methods for Nonlinear Estimation. Thesis No. 1047, 2003.

F. Gunnarsson: On Modeling and Control of Network Queue Dynamics. Thesis No. 1048, 2003.

S. Björklund: A Survey and Comparison of Time-Delay Estimation Methods in Linear Systems. Thesis No. 1061, 2003. 
M. Gerdin: Parameter Estimation in Linear Descriptor Systems. Thesis No. 1085, 2004.

A. Eidehall: An Automotive Lane Guidance System. Thesis No. 1122, 2004.

E. Wernholt: On Multivariable and Nonlinear Identification of Industrial Robots. Thesis No. 1131, 2004.

J. Gillberg: Methods for Frequency Domain Estimation of Continuous-Time Models. Thesis No. 1133, 2004.

G. Hendeby: Fundamental Estimation and Detection Limits in Linear Non-Gaussian Systems. Thesis No. 1199, 2005.

D. Axehill: Applications of Integer Quadratic Programming in Control and Communication. Thesis No. 1218, 2005.

J. Sjöberg: Some Results On Optimal Control for Nonlinear Descriptor Systems. Thesis No. 1227, 2006.

D. Törnqvist: Statistical Fault Detection with Applications to IMU Disturbances. Thesis No. 1258, 2006.

H. Tidefelt: Structural algorithms and perturbations in differential-algebraic equations. Thesis No. 1318, 2007.

S. Moberg: On Modeling and Control of Flexible Manipulators. Thesis No. 1336, 2007.

J. Wallén: On Kinematic Modelling and Iterative Learning Control of Industrial Robots. Thesis No. 1343, 2008.

J. Harju Johansson: A Structure Utilizing Inexact Primal-Dual Interior-Point Method for Analysis of Linear Differential Inclusions. Thesis No. 1367, 2008.

J. D. Hol: Pose Estimation and Calibration Algorithms for Vision and Inertial Sensors. Thesis No. 1370, 2008.

H. Ohlsson: Regression on Manifolds with Implications for System Identification. Thesis No. 1382, 2008.

D. Ankelhed: On low order controller synthesis using rational constraints. Thesis No. 1398, 2009.

P. Skoglar: Planning Methods for Aerial Exploration and Ground Target Tracking. Thesis No. 1420, 2009.

C. Lundquist: Automotive Sensor Fusion for Situation Awareness. Thesis No. 1422, 2009.

C. Lyzell: Initialization Methods for System Identification. Thesis No. 1426, 2009.

R. Falkeborn: Structure exploitation in semidefinite programming for control. Thesis No. 1430, 2010.

D. Petersson: Nonlinear Optimization Approaches to $\mathcal{H}_{2}$-Norm Based LPV Modelling and Control. Thesis No. 1453, 2010.

Z. Sjanic: Navigation and SAR Auto-focusing in a Sensor Fusion Framework. Thesis No. 1464, 2011.

K. Granström: Loop detection and extended target tracking using laser data. Thesis No. 1465, 2011.

J. Callmer: Topics in Localization and Mapping. Thesis No. 1489, 2011.

F. Lindsten: Rao-Blackwellised particle methods for inference and identification. Thesis No. 1480, 2011.

M. Skoglund: Visual Inertial Navigation and Calibration. Thesis No. 1500, 2011.

S. Khoshfetrat Pakazad: Topics in Robustness Analysis. Thesis No. 1512, 2011.

P. Axelsson: On Sensor Fusion Applied to Industrial Manipulators. Thesis No. 1511, 2011.

A. Carvalho Bittencourt: On Modeling and Diagnosis of Friction and Wear in Industrial Robots. Thesis No. 1516, 2012.

P. Rosander: Averaging level control in the presence of frequent inlet flow upsets. Thesis No. 1527, 2012. 
N. Wahlström: Localization using Magnetometers and Light Sensors. Thesis No. 1581, 2013.

R. Larsson: System Identification of Flight Mechanical Characteristics. Thesis No. 1599, 2013.

Y. Jung: Estimation of Inverse Models Applied to Power Amplifier Predistortion. Thesis No. 1605, 2013.

M. Syldatk: On Calibration of Ground Sensor Networks. Thesis No. 1611, 2013.

M. Roth: Kalman Filters for Nonlinear Systems and Heavy-Tailed Noise. Thesis No. 1613, 2013.

D. Simon: Model Predictive Control in Flight Control Design - Stability and Reference Tracking. Thesis No. 1642, 2014.

J. Dahlin: Sequential Monte Carlo for inference in nonlinear state space models. Thesis No. 1652, 2014.

M. Kok: Probabilistic modeling for positioning applications using inertial sensors. Thesis No. 1656, 2014.

J. Linder: Graybox Modelling of Ships Using Indirect Input Measurements. Thesis No. 1681, 2014.

G. Mathai: Direction of Arrival Estimation of Wideband Acoustic Wavefields in a Passive Sensing Environment. Thesis No. 1721, 2015.

I. Nielsen: On Structure Exploiting Numerical Algorithms for Model Predictive Control. Thesis No. 1727, 2015.

C. Veibäck: Tracking of Animals Using Airborne Cameras. Thesis No. 1761, 2016.

N. Evestedt: Sampling Based Motion Planning for Heavy Duty Autonomous Vehicles. Thesis No. 1762, 2016.

H. Nyqvist: On Pose Estimation in Room-Scaled Environments. Thesis No. 1765, 2016.

Y. Zhao: Position Estimation in Uncertain Radio Environments and Trajectory Learning. Thesis No. 1772, 2017.

P. Kasebzadeh: Parameter Estimation for Mobile Positioning Applications. Thesis No. 1786, 2017.

K. Radnosrati: On Timing-Based Localization in Cellular Radio Networks. Thesis No. 1808, 2018.

G. Lindmark: Methods and Algorithms for Control Input Placement in Complex Networks. Thesis No. 1814, 2018.

M. Lindfors: Frequency Tracking for Speed Estimation. Thesis No. 1815, 2018.

D. Ho: Some results on closed-loop identification of quadcopters. Thesis No. 1826, 2018.

O. Ljungqvist: On motion planning and control for truck and trailer systems. Thesis No. 1832, 2019.

P. Boström-Rost: On Informative Path Planning for Tracking and Surveillance. Thesis No. 1838, 2019.

K. Bergman: On Motion Planning Using Numerical Optimal Control. Thesis No. 1843, 2019.

M. Klingspor: Low-rank optimization in system identification. Thesis No. 1855, 2019.

A. Bergström: Timing-Based Localization using Multipath Information. Thesis No. 1867, 2019.

F. Ljungberg: Estimation of Nonlinear Greybox Models for Marine Applications. Thesis No. 1880, 2020. 


\section{FACULTY OF SCIENCE AND ENGINEERING}

Linköping studies in science and technology. Licentiate Thesis No. 1894, 2020 Department of Electrical Engineering

Linköping University

SE-581 83 Linköping, Sweden

www.liu.se 